\title{
Homogenized Model of Two-Phase Flow with Local Nonequilibrium in Double Porosity Media
}

\author{
Brahim Amaziane, ${ }^{1}$ Mikhail Panfilov, ${ }^{2,3}$ and Leonid Pankratov ${ }^{1,4}$ \\ ${ }^{1}$ Laboratoire de Mathématiques et leurs Applications, CNRS-UMR 5142, Université de Pau, Pau, France \\ ${ }^{2}$ Institut Elie Cartan de Lorraine, CNRS-UMR 7502/Université de Lorraine, Nancy, France \\ ${ }^{3}$ Institut Jean le Rond d'Alembert, CNRS-UMR 7190/Sorbonne Universités, Paris, France \\ ${ }^{4}$ Laboratory of Fluid Dynamics and Seismic (RAEP 5 Top 100), Moscow Institute of Physics and Technology, 9 Institutskiy Per., \\ Dolgoprudny, Moscow Region 141700, Russia \\ Correspondence should be addressed to Mikhail Panfilov; mikhail.panfilov@univ-lorraine.fr
}

Received 16 March 2016; Revised 15 June 2016; Accepted 30 June 2016

Academic Editor: Prabir Daripa

Copyright (C) 2016 Brahim Amaziane et al. This is an open access article distributed under the Creative Commons Attribution License, which permits unrestricted use, distribution, and reproduction in any medium, provided the original work is properly cited.

We consider two-phase flow in a heterogeneous porous medium with highly permeable fractures and low permeable periodic blocks. The flow in the blocks is assumed to be in local capillary disequilibrium and described by Barenblatt's relaxation relationships for the relative permeability and capillary pressure. It is shown that the homogenization of such equations leads to a new macroscopic model that includes two kinds of long-memory effects: the mass transfer between the blocks and fractures and the memory caused by the microscopic Barenblatt disequilibrium. We have obtained a general relationship for the double nonequilibrium capillary pressure which represents great interest for applications. Due to the nonlinear coupling and the nonlocality in time, the macroscopic model remains incompletely homogenized in general case. The completely homogenized model was obtained for two different regimes. The first case corresponds to a linearized flow in the blocks. In the second case, we assume a low contrast in the block-fracture permeability. Numerical results for the two-dimensional problem are presented for two test cases to demonstrate the effectiveness of the methodology.

\section{Introduction}

The classical theory of multiphase flow of liquids and gases through porous media is based on the hypothesis of the local thermodynamic equilibrium. This means that, in each representative elementary volume (REV) of a porous medium, the thermobaric, the capillary, and the chemical equilibrium between the fluid phases is established instantly. Until the phases form a small-scale mixture in porous space with the characteristic size of droplets (bubbles, ganglions, menisci, films, etc.) about the radius of pores, the thermobaric and chemical equilibrium between two phases is totally acceptable, which is not however the case of the capillary equilibrium.

The capillary equilibrium determines a special distribution of two phases in the pore network such that the resulting capillary force is nil. According to the classical theory, such an equilibrium phase distribution is reached when the wetting fluid prefers to occupy narrow pores, while the nonwetting fluid is confined in large pores. However if we displace oil by water, the displaced oil (nonwetting) ahead of the front occupies all the pores including narrow, while the injected water (wetting) behind the front moves in all the pores including the large ones. Such a fluid distribution does not correspond to the equilibrium state; then a relaxation process spontaneously starts in the medium and tends to redistribute the two fluids over the pore network. This redistribution consists of the rearrangement of phase clusters, implies the fluid replacement to long distances, which concerns the overall fluid, and, consequently, is nonlocal. The redistribution of phases represents the intrinsic flow of both phases caused by the capillary forces (applied to the curved interfaces between two phases) and slowed by the friction against the pore walls. Such a flow occurs within a pore network 
having usually a low connectivity, which reduces the rate of phase redistribution. The lower the medium permeability, the higher the friction, and the lower the connectivity of the pore network. Consequently, the redistribution time in low permeable media is expected to be large, such that the hypothesis of the local capillary equilibrium can no longer be accepted.

The modeling of such redistribution/relaxation was introduced first by Barenblatt [1] with modifications in [2, 3] and developed further by a number of authors. In [1], it is assumed that the relative permeability $\left(k_{r w}, k_{r o}\right)$ and the capillary pressure $\left(p_{c}\right)$ depend not on the true instantaneous saturation $S$, but on an effective saturation $\boldsymbol{\eta}$ that corresponds to the equilibrium value that might be reached by the system after a phase redistribution in space:

$$
\begin{aligned}
\boldsymbol{\eta} & :=S+\tau \frac{\partial S}{\partial t}, \\
p_{c} & =p_{c}(\boldsymbol{\eta}), \\
k_{r \alpha} & =k_{r \alpha}(\boldsymbol{\eta}), \quad \alpha=w, o,
\end{aligned}
$$

where $\tau$ is the redistribution time. Note that $\partial S / \partial t$ is the rate of the phase redistribution; therefore $\tau(\partial S / \partial t)$ means the saturation variation from a nonequilibrium value up to the equilibrium one.

Theoretical justifications of relationship (1) have been obtained by several authors using the approach of the nonequilibrium thermodynamics: first by Nikolaevskii et al. [4], published only in Russian, by Marle in [5], and later by Hassanizadeh and Gray [6]. The method consists in formulating the relationship for the entropy production as a bilinear form made up of the products between thermodynamic forces and fluxes and assuming Onsager's linear relationships between the forces and fluxes of the same tensor dimension.

Let us now consider a double porosity medium, which represents a connected network of highly permeable porous channels ("the fractures") and very low permeable inclusions ("the blocks"). As mentioned above, the capillary nonequilibrium should be retained in the blocks, which is the objective of the present paper.

The two-phase flow in double porosity media at local equilibrium is well studied; see, for example, [7-9]. It was shown that, in such systems, despite the local equilibrium, another nonequilibrium phenomenon appears on the macroscale, which is caused by the high delay in capillary redistribution of the fluids between blocks and fractures. This disequilibrium is described in terms of long-memory operators, which appear in the macroscopic model of flow. In $[8,9]$, the obtained general macroscopic model was not completely homogenized. A completely homogenized model was obtained in the same papers $[8,9]$ by the linearization of the local problem in the block, which allowed obtaining an explicit integrodifferential expression for the memory. Consequently the differential microscopic flow equations transform into integrodifferential macroscopic equations.

Another completely homogenized model (always without Barenblatt's nonequilibrium) was obtained in $[10,11]$ for the case of a lower contrast between the block and fracture permeability. This case leads to a lower degree of the disequilibrium, described by a model with short memory. The macroscopic model was completely homogenized without any linearization.

If now we introduce the microscopic Barenblatt capillary nonequilibrium in a double porosity medium, then the microscopic and the macroscopic nonequilibrium effects will interact with each other. The physical mechanism of this interaction is still unclear, and its mathematical model is unknown. The analysis of this interaction is the main objective of the present paper. More precisely, our aim is to develop a macroscopic model for two-phase incompressible flow in a double porosity medium including the local nonequilibrium.

The two-phase flow in double porosity medium with local nonequilibrium has been studied in [12] where upscaling was performed numerically.

Usually the local capillary nonequilibrium is introduced through the dynamic capillary pressure relationship. Another approach is based on Kondaurov's phenomenology developed for viscoelasticity. This approach was applied to twophase flow in double porosity media in $[13,14]$.

Mathematical study of the immiscible two-phase flow with local nonequilibrium in porous media concerns, for the moment, only homogeneous media. The local nonequilibrium is introduced through the dynamic capillary pressuresaturation relationship. Existence and uniqueness results can be found in [15-18], traveling wave solutions have been obtained in [19-21], and the numerical analysis of the nonequilibrium models is presented in $[22,23]$.

In this paper, the homogenization method is used to derive the upscaled model for two-phase flow with local Barenblatt's nonequilibrium in double porosity media with periodic microstructure. More precisely, we have obtained the following new results:

(i) a completely homogenized model for the case of a linearized flow in blocks;

(ii) a completely homogenized model for the case of low contrast in the block-fracture properties;

(iii) an explicit relationship for calculating the memory functions and the relaxation times;

(iv) the qualitative effects of the nonequilibrium effects described by double memory operators (double convolution integrals).

The rest of the paper is organized as follows. In the next section, we formulate the two-phase flow problem with local nonequilibrium effects on the microscale and formulate the main assumptions on the data. Section 3 is devoted to the presentation of the homogenization method. First, in Section 3.1 we present the two-scale asymptotic expansions. Secondly, the macroscopic variables are defined in Section 3.2. Thirdly, in Section 3.3 we obtain a homogenized model. Finally, in Section 3.4 we derive, in addition, a relationship between the average capillary pressures in blocks and fractures. In Section 4 we are dealing with the first completely homogenized model obtained for the case of a linearized cell problem in the blocks. The second completely homogenized model is 
formulated in Section 5 for the case of low contrast in blockfracture properties. In Section 6, we exhibit the numerical results for two test cases. We present numerical simulations to analyze the impact of the nonequilibrium effects for twophase flow in a fractured reservoir. Additional conclusions are drawn in Section 7.

\section{Formulation of the Problem at the Microscale and Main Assumptions}

2.1. Medium Geometry and Properties. We consider twophase immiscible flow of oil and water at the Darcy scale in an inhomogeneous porous medium consisting of two types of rocks having different porosities and permeability. The heterogeneities are organized in the form of a periodic system of low permeable blocks immersed into a connected highly permeable subdomain called "fractures." The heterogeneity size is small with respect to the entire domain size. Such a system has an intrinsic macroscopic behavior, independent of the boundary conditions. Consequently, we will distinguish two different scales of consideration:

(i) the microscale: the mathematical point corresponds to an elementary representative volume of porous medium; the microscale flow is described by Darcy's law;

(ii) the macroscale: the mathematical point corresponds to a period of medium heterogeneity. The macroscopic flow equations may be obtained by the homogenization of the microscale equations.

To describe the microscopic geometry, we will introduce the following notations: the overall domain $\Omega \subset \mathbb{R}^{d}(d=2,3)$ is bounded and connected and has periodic structure. The heterogeneity period $\varepsilon$ is a small parameter. The low permeable blocks are $\Omega_{\mathrm{m}}^{\varepsilon}$, and the connected highly permeable medium (the fracture) is $\Omega_{\mathrm{f}}^{\varepsilon}$. The interface between the blocks and the fracture is $\Gamma_{\mathrm{fm}}^{\varepsilon}$. Let $Y:=(0,1)^{d}$ be the period of the medium extended by $\varepsilon^{-1}$ times. Then $Y_{\mathrm{m}}, Y_{\mathrm{f}}$, and $\Gamma_{\mathrm{fm}}$ are the images of $\Omega_{\mathrm{m}}^{\varepsilon}, \Omega_{\mathrm{f}}^{\varepsilon}$, and $\Gamma_{\mathrm{fm}}^{\varepsilon}$ respectively; see Figure 1 . We also introduce the notations: $\Omega_{T}:=\Omega \times(0, T)$ and $\Omega_{\ell, T}^{\varepsilon}:=\Omega_{\ell, T}^{\varepsilon} \times(0, T)$, for $\ell=\mathrm{f}, \mathrm{m}$. Note that the fractures are not an empty space but represent a porous medium with permeability much higher than that of the blocks.

The fractures are not thin; that is, their thickness is not an additional small parameter, as in [24].

The double porosity medium is defined through the following properties of the porosity $\phi^{\varepsilon}(x)$ and the absolute permeability tensor $K^{\varepsilon}(x)$ :

$$
\begin{aligned}
\phi^{\varepsilon}(x) & := \begin{cases}\phi_{\mathrm{f}} & \text { in fractures, } \\
\phi_{\mathrm{m}} & \text { in blocks, }\end{cases} \\
K^{\varepsilon}(x) & := \begin{cases}K_{\mathrm{f}} \rrbracket & \text { in fractures, } \\
\varepsilon^{2} K_{\mathrm{m}} \rrbracket & \text { in blocks, }\end{cases}
\end{aligned}
$$

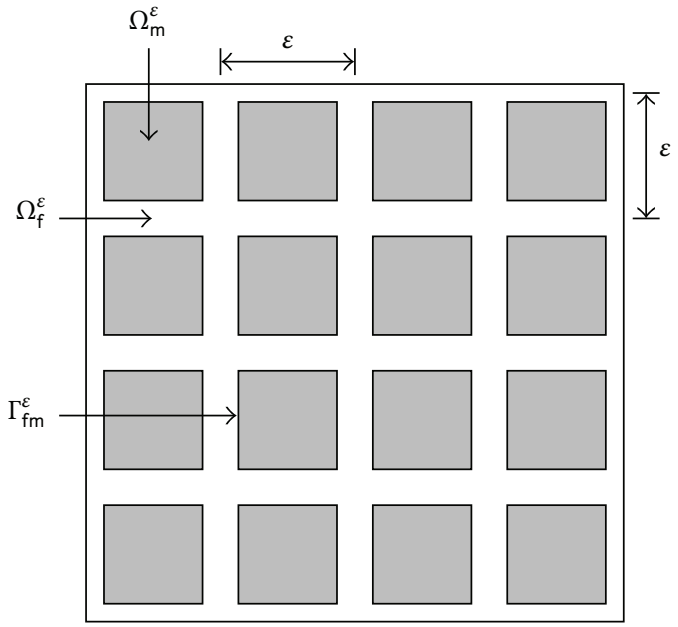

(a)

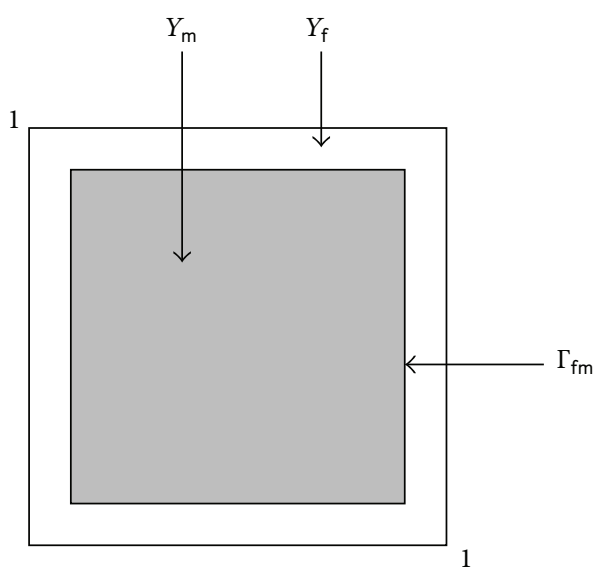

(b)

FIgURE 1: (a) The domain $\Omega$ with the mesostructure. (b) The reference cell $Y$.

where the values $0<\phi_{\mathrm{f}}, \phi_{\mathrm{m}}<1,0<K_{\mathrm{f}}, K_{\mathrm{m}}<K_{\max }<+\infty$ are positive constants that do not depend on $\varepsilon$; and $\square$ is the identity matrix.

2.2. Microscale Nonequilibrium Effects. We assume that the flow in the fractures is in equilibrium, while it is in local disequilibrium in the blocks. This means that the relative permeability and the capillary pressures may be considered as functions only of the local saturation in fractures, while in blocks they depend on a nonlocal functional of saturation which keeps the memory of the process. We adopt the model of Barenblatt with short memory, by introducing the functional $\boldsymbol{\eta}_{\mathrm{m}}^{\varepsilon}\left(S_{\mathrm{m}}^{\varepsilon}, \partial_{t} S_{\mathrm{m}}^{\varepsilon}\right)$, called the efficient saturation and defined similar to (1):

$$
\boldsymbol{\eta}_{\mathrm{m}}^{\varepsilon}=S_{\mathrm{m}}^{\varepsilon}+\tau_{\mathrm{m}} \frac{\partial S_{\mathrm{m}}^{\varepsilon}}{\partial t},
$$

where $\tau_{\mathrm{m}}$ is the relaxation time in blocks $\Omega_{\mathrm{m}}^{\varepsilon}$, and $S_{\mathrm{m}}^{\varepsilon}$ is the water saturation in blocks.

We assume that the relative permeability and the capillary pressure in blocks depend only on this functional. The degree 
of the disequilibrium is determined by the relaxation time $\tau_{\mathrm{m}}$ : the larger $\tau_{\mathrm{m}}$, the higher disequilibrium in the system.

The relaxation time in fractures is much lower than in blocks, and, consequently, we can assume the local equilibrium in fractures.

2.3. Microscale Flow Equations. Let us introduce the following notations, for $\ell=\mathrm{f}, \mathrm{m}$ :

(i) $S_{\ell}^{\varepsilon}=S_{\ell, w}^{\varepsilon}$ is the water saturation in the medium $\Omega_{\ell}^{\varepsilon}$ (the oil saturation is then $1-S_{\ell}^{\varepsilon}$ );

(ii) $p_{\ell, w}^{\varepsilon}(x, t)$ and $p_{\ell, o}^{\varepsilon}(x, t)$ are the pressures of water and oil in medium $\Omega_{\ell}^{\varepsilon}$;

(iii) $k_{\mathrm{f}, w}\left(S_{\mathrm{f}}^{\varepsilon}\right)$ and $k_{\mathrm{f}, o}\left(S_{\mathrm{f}}^{\varepsilon}\right)$ are the relative permeability of water and of oil in fractures $\Omega_{f}^{\varepsilon}$, and they are given functions and depend only on the local saturation;

(iv) $k_{\mathrm{m}, w}\left(\boldsymbol{\eta}_{\mathrm{m}}^{\varepsilon}\right)$ and $k_{\mathrm{m}, o}\left(\boldsymbol{\eta}_{\mathrm{m}}^{\varepsilon}\right)$ are the relative permeability of water and of oil in blocks $\Omega_{\mathrm{m}}^{\varepsilon}$, and they are given functions and depend only on the nonlocal functional $\boldsymbol{\eta}_{\mathrm{m}}^{\varepsilon}$

(v) $\lambda_{\mathrm{f}, j}\left(S_{\mathrm{f}}^{\varepsilon}\right)=k_{\mathrm{f}, j}\left(S_{\mathrm{f}}^{\varepsilon}\right) / \mu_{j}$ and $\lambda_{\mathrm{m}, j}\left(\boldsymbol{\eta}_{\mathrm{m}}^{\varepsilon}\right)=k_{\mathrm{m}, j}\left(\boldsymbol{\eta}_{\mathrm{m}}^{\varepsilon}\right) / \mu_{j}$ are the phase mobility $(j=w, o)$;

(vi) $\mu_{w}$ and $\mu_{o}$ are the dynamic viscosities which are assumed to be positive constants;

(vii) $P_{\mathrm{f}, \mathrm{c}}\left(S_{\mathrm{f}}^{\varepsilon}\right)$ and $P_{\mathrm{m}, \mathrm{c}}\left(\boldsymbol{\eta}_{\mathrm{m}}^{\varepsilon}\right)$ are the capillary pressures in fractures and in blocks.

In what follows, we assume that the capillary pressure functions $P_{\mathrm{f}, \mathrm{c}}\left(S_{\mathrm{f}}^{\varepsilon}\right), P_{\mathrm{m}, c}\left(\boldsymbol{\eta}_{\mathrm{m}}^{\varepsilon}\right)$ are positive decreasing Lipschitz functions of their arguments. We also assume that there exist constants $m, M \in(0, \infty)$ such that the relative permeability satisfies the bounds:

$$
\begin{aligned}
0<m \leqslant k_{\beta, j}(\varsigma) \leqslant & \\
& \forall \varsigma \in[0,1](\beta=\{\mathrm{f}, \mathrm{m}\} ; j=\{w, o\}) .
\end{aligned}
$$

2.4. Microscale Flow Equations. For the sake of simplicity, we neglect the gravitational effects and the source/sink terms.

2.4.1. Mass Balance in Fractures. The mass conservation of each phase can be written in the framework of the standard Darcy-Muskat equations [25-28]. In $\Omega_{\mathrm{f}, T}^{\varepsilon}$ :

$$
\begin{aligned}
\phi_{\mathrm{f}} \frac{\partial S_{\mathrm{f}}^{\varepsilon}}{\partial t}-\operatorname{div}\left\{K_{\mathrm{f}}(x) \lambda_{\mathrm{f}, w}\left(S_{\mathrm{f}}^{\varepsilon}\right) \nabla p_{\mathrm{f}, w}^{\varepsilon}\right\} & =0 ; \\
-\phi_{\mathrm{f}} \frac{\partial S_{\mathrm{f}}^{\varepsilon}}{\partial t}-\operatorname{div}\left\{K_{\mathrm{f}}(x) \lambda_{\mathrm{f}, o}\left(S_{\mathrm{f}}^{\varepsilon}\right) \nabla p_{\mathrm{f}, o}^{\varepsilon}\right\} & =0 ; \\
p_{\mathrm{f}, o}^{\varepsilon}-p_{\mathrm{f}, w}^{\varepsilon} & =P_{\mathrm{f}, c}\left(S_{\mathrm{f}}^{\varepsilon}\right) .
\end{aligned}
$$

2.4.2. Mass Balance in Blocks. We apply the Darcy-Muskat model with Barenblatt's capillary nonequilibrium [29-31]. In $\Omega_{\mathrm{m}, T}^{\varepsilon}$ :

$$
\begin{aligned}
& \phi_{\mathrm{m}} \frac{\partial S_{\mathrm{m}}^{\varepsilon}}{\partial t}-\varepsilon^{2} \operatorname{div}\left\{K_{\mathrm{m}}(x) \lambda_{\mathrm{m}, w}\left(\boldsymbol{\eta}_{\mathrm{m}}^{\varepsilon}\right) \nabla p_{\mathrm{m}, w}^{\varepsilon}\right\}=0 \\
& -\phi_{\mathrm{m}} \frac{\partial S_{\mathrm{m}}^{\varepsilon}}{\partial t}-\varepsilon^{2} \operatorname{div}\left\{K_{\mathrm{m}}(x) \lambda_{\mathrm{m}, o}\left(\boldsymbol{\eta}_{\mathrm{m}}^{\varepsilon}\right) \nabla p_{\mathrm{m}, o}^{\varepsilon}\right\}=0 \\
& p_{\mathrm{m}, o}^{\varepsilon}-p_{\mathrm{m}, w}^{\varepsilon}=P_{\mathrm{m}, c}\left(\boldsymbol{\eta}_{\mathrm{m}}^{\varepsilon}\right) \\
& \boldsymbol{\eta}_{\mathrm{m}}^{\varepsilon}=S_{\mathrm{m}}^{\varepsilon}+\tau_{\mathrm{m}} \frac{\partial S_{\mathrm{m}}^{\varepsilon}}{\partial t}
\end{aligned}
$$

2.4.3. Conditions at the Block-Fracture Interface. We assume the continuity of the phase fluxes and pressures at the interface $\Gamma_{\mathrm{fm}}^{\mathcal{E}}$ :

$$
\begin{aligned}
& K_{\mathrm{f}} \lambda_{\mathrm{m}, j} \nabla p_{\mathrm{m}, j}^{\varepsilon} \cdot \vec{v}=\varepsilon^{2} K_{\mathrm{m}} \lambda_{\mathrm{f}, j} \nabla p_{\mathrm{f}, j}^{\varepsilon} \cdot \vec{\nu}, \\
& p_{\mathrm{f}, j}^{\varepsilon}=p_{\mathrm{m}, j}^{\varepsilon}, j=w, o,
\end{aligned}
$$

where $\vec{v}$ is the unit outer normal on $\Gamma_{\mathrm{fm}}^{\varepsilon}$.

Note that the continuity of phase pressures leads to the continuity of the capillary pressure. In several situations the capillary pressure may be discontinuous, which is the case of the so-called capillary trapping of the nonwetting phase in low permeable blocks $[32,33]$. These effects are not analyzed in the present paper.

2.4.4. Initial Conditions. The initial condition in the fracture system read:

$$
S_{\mathrm{f}}^{\varepsilon}(x, 0)=S_{\mathrm{f}}^{\text {init }}(x) \text { in } \Omega_{\mathrm{f}}^{\varepsilon} \text {. }
$$

In the present paper we consider only the situation of the initial equilibrium state, when $\boldsymbol{\eta}_{\mathrm{m}}^{\text {init }}(x)=S_{\mathrm{m}}^{\text {init }}(x)$. In more general case the dependence of $\boldsymbol{\eta}_{\mathrm{m}}^{\text {init }}$ on $S_{\mathrm{m}}^{\text {init }}$ is determined from the last equation in (7), [31]. Then the initial conditions in the matrix part read:

$$
\begin{aligned}
& S_{\mathrm{m}}^{\varepsilon}(x, 0)=S_{\mathrm{m}}^{\text {init }}(x), \\
& \boldsymbol{\eta}_{\mathrm{m}}^{\varepsilon}(x, 0)=\boldsymbol{\eta}_{\mathrm{m}}^{\text {init }}(x)=S_{\mathrm{m}}^{\text {init }}(x)
\end{aligned}
$$

$$
\text { in } \Omega_{\mathrm{m}}^{\varepsilon} \text {. }
$$

These equations will be called "the microscopic flow model." The conditions at the reservoir boundary are assumed to not influence the homogenized model.

\section{Upscaled Model}

In this section we present the upscaled model derived by the two-scale asymptotic expansions. The technique of derivation is presented in Appendix.

3.1. Two-Scale Asymptotic Expansions. We assume that the solutions to problem (6)-(8) depend on the space variable 
in the following sense: they are functions of the macroscopic (or "slow") variable $x \in \Omega$ and the microscopic (or "fast") variable $y \in Y$. The macroscopic and microscopic scales are related by the small parameter $\varepsilon$ up to a translation as $y=x / \varepsilon$. This implies that

$$
\frac{\partial}{\partial x_{i}}:=\frac{\partial}{\partial x_{i}}+\frac{1}{\varepsilon} \frac{\partial}{\partial y_{i}} \quad(i=1, \ldots, d)
$$

or equivalently $\nabla:=\nabla_{x}+\varepsilon^{-1} \nabla_{y}$,

where $\nabla_{x}, \nabla_{y}$ are the gradients with respect to the variables $x, y$.

Note that if a function $\phi^{\varepsilon}(x)=\phi(x / \varepsilon)$ is $\varepsilon Y$-periodic, then $\phi^{\varepsilon}(x)=\phi(y)$.

We are interested in finding the set $\left\langle p_{\mathrm{f}, w}^{\varepsilon}, p_{\mathrm{f}, o}^{\varepsilon}, p_{\mathrm{m}, w}^{\varepsilon}\right.$, $\left.p_{\mathrm{m}, o}^{\varepsilon}, S_{\mathrm{f}}^{\varepsilon}, S_{\mathrm{m}}^{\varepsilon}, \boldsymbol{\eta}_{\mathrm{m}}^{\varepsilon}\right\rangle$, which is the solution to (6)-(8) having the following asymptotic expansion:

$$
\begin{aligned}
p_{\mathrm{f}, j}^{\varepsilon} & =P_{\mathrm{f}, j}(x, t)+\varepsilon p_{\mathrm{f}, j}^{(1)}(x, y, t)+\varepsilon^{2} \cdots, \quad j=w, o, \\
p_{\mathrm{m}, j}^{\varepsilon} & =P_{\mathrm{m}, j}(x, y, t)+\varepsilon p_{\mathrm{m}, j}^{(1)}(x, y, t)+\varepsilon^{2} \cdots,
\end{aligned} \quad j=w, o,
$$

where all the coefficients of these expansions are $Y$-periodic in $y$.

For the phase mobility, we obtain the asymptotic expansion (for $j=w, o)$ :

$$
\begin{gathered}
\lambda_{\mathrm{f}, j}\left(S_{\mathrm{f}}^{\varepsilon}\right)=\lambda_{\mathrm{f}, j}\left(S_{\mathrm{f}}\right)+\varepsilon \lambda_{\mathrm{f}, j}^{\prime}\left(S_{\mathrm{f}}\right) S_{\mathrm{f}}^{(1)}+\cdots, \\
\lambda_{\mathrm{m}, j}\left(\boldsymbol{\eta}_{\mathrm{m}}^{\varepsilon}\right)=\lambda_{\mathrm{m}, j}\left(\boldsymbol{\eta}_{\mathrm{m}}\right)+\varepsilon \lambda_{\mathrm{m}, j}^{\prime}\left(\boldsymbol{\eta}_{\mathrm{m}}\right) \boldsymbol{\eta}_{\mathrm{m}}^{(1)}+\cdots .
\end{gathered}
$$

Note that the first terms $P_{\mathrm{f}, w}, P_{\mathrm{f}, o}, S_{\mathrm{f}}$ do not depend on the fast variable $y$, which is explained by the fact that the saturation field in fractures is instantaneously established and, consequently, is uniform within a cell; see, for instance, $[7,34]$.

3.2. Macroscopic Variables. The physical meaning of the first terms of the asymptotic expansions is

(i) $S_{f}(x, t), P_{w}(x, t)$, and $P_{o}(x, t)$ are the macroscopic water saturation, water pressure, and oil pressure in fractures;

(ii) $s_{\mathrm{m}}(x, y, t), p_{w}(x, y, t)$, and $p_{o}(x, y, t)$ are the nonhomogenized first terms of the asymptotic expansions for the local water saturation, water pressure, and oil pressure in blocks;

(iii) $\boldsymbol{\eta}_{\mathrm{m}}(x, y, t)$ is the nonhomogenized first term for the nonlocal Barenblatt saturation in blocks;

(iv) $\left|Y_{\ell}\right|$ is the volume of the domain $Y_{\ell}(\ell=\mathrm{f}, \mathrm{m})$; (v) $\langle u\rangle_{\ell}=\left(1 /\left|Y_{\ell}\right|\right) \int_{Y_{\ell}} u(y) d y(\ell=\mathrm{f}, \mathrm{m})$;

(vi) $\phi^{\star}=\phi_{\mathrm{f}}\left|Y_{\mathrm{f}}\right|$ is the effective porosity of fractures;

(vii) $\mathbb{K}_{i j}^{\star}(x)=K_{\mathrm{f}}\left|Y_{\mathrm{f}}\right|\left\langle\left[\nabla_{y} \zeta_{i}+\vec{e}_{i}\right]\left[\nabla_{y} \zeta_{j}+\vec{e}_{j}\right]\right\rangle_{\mathrm{f}}, 1 \leq i, j \leq d$, is the macroscopic tensor of the absolute permeability, homogenized over the fractures, where $\zeta_{j}, 1 \leq j \leq d$, is a solution of the following auxiliary cell problem:

$$
\begin{gathered}
\Delta_{y} \zeta_{j}=0 \quad \text { in } Y_{\mathrm{f}} ; \\
\nabla_{y} \zeta_{j} \cdot \vec{v}_{y}=-\vec{e}_{j} \cdot \vec{v}_{y} \quad \text { on } \Gamma_{\mathrm{fm}}, \\
\zeta_{j}(y) \text { is } Y \text {-periodic, }
\end{gathered}
$$

where $\vec{e}_{1}, \ldots, \vec{e}_{d}$ is the canonical basis of $\mathbb{R}^{d}$.

3.3. Macroscopic Model. The technique of homogenization is presented in Appendix.

The homogenized model reads in $\Omega_{T}$

$$
\begin{gathered}
\phi^{\star} \frac{\partial S_{\mathrm{f}}}{\partial t}-\operatorname{div}_{x}\left\{\mathbb{K}^{\star}(x) \lambda_{\mathrm{f}, w}\left(S_{\mathrm{f}}\right) \nabla P_{w}\right\} \\
=-\phi_{\mathrm{m}}\left|Y_{\mathrm{m}}\right| \frac{\partial\left\langle s_{\mathrm{m}}\right\rangle_{\mathrm{m}}}{\partial t}=Q_{w}, \\
-\phi^{\star} \frac{\partial S_{\mathrm{f}}}{\partial t}-\operatorname{div}_{x}\left\{\mathbb{K}^{\star}(x) \lambda_{\mathrm{f}, o}\left(S_{\mathrm{f}}\right) \nabla P_{o}\right\} \\
=\phi_{\mathrm{m}}\left|Y_{\mathrm{m}}\right| \frac{\partial\left\langle s_{\mathrm{m}}\right\rangle_{\mathrm{m}}}{\partial t}=-Q_{w}, \\
P_{o}-P_{w}=P_{\mathrm{f}, c}\left(S_{\mathrm{f}}\right)
\end{gathered}
$$

with the initial condition $S_{f}(x, 0)=S_{f}^{\text {init }}(x)$.

This model is determined through the function $s_{\mathrm{m}}(x, y, t)$ that is the local saturation in blocks, which is a nonlocal (in time) functional of the effective saturation $\boldsymbol{\eta}_{\mathrm{m}}$ :

$$
\begin{aligned}
s_{\mathrm{m}}(x, y, t)= & \mathcal{S}_{\mathrm{m}}\left[\boldsymbol{\eta}_{\mathrm{m}}\right] \\
= & S_{\mathrm{m}}^{\text {init }}(x) e^{-t / \tau_{\mathrm{m}}} \\
& +\frac{1}{\tau_{\mathrm{m}}}\left(e^{-t / \tau_{\mathrm{m}}} \star \boldsymbol{\eta}_{\mathrm{m}}\right)(x, y, t),
\end{aligned}
$$

where $(f \star g)(t)=\int_{0}^{t} f(t-\varsigma) g(\varsigma) d \varsigma$.

This is the explicit solution to (4) reformulated for the zero terms of the asymptotic expansion (12).

The function $\boldsymbol{\eta}_{\mathrm{m}}$, in turn, is the solution to the following problem within a block $y \in Y_{\mathrm{m}}$ :

$$
\begin{array}{ll}
\phi_{\mathrm{m}} \frac{\partial}{\partial t} \mathcal{S}_{\mathrm{m}}\left[\boldsymbol{\eta}_{\mathrm{m}}\right]-\operatorname{div}_{y}\left(K_{\mathrm{m}} \alpha_{\mathrm{m}}\left(\boldsymbol{\eta}_{\mathrm{m}}\right) \nabla_{y} \boldsymbol{\eta}_{\mathrm{m}}\right)=0 & \text { in } Y_{\mathrm{m}} ; \\
\boldsymbol{\eta}_{\mathrm{m}}(x, y, t)=\mathscr{P}\left(S_{\mathrm{f}}(x, t)\right) & \\
\text { on } \Gamma_{\mathrm{fm}} \text { with } \mathscr{P}\left(S_{\mathrm{f}}\right)=\left(P_{\mathrm{m}, \mathrm{c}}^{-1} \circ P_{\mathrm{f}, \mathrm{c}}\right)\left(S_{\mathrm{f}}\right)
\end{array}
$$


with the initial condition: $\boldsymbol{\eta}_{\mathrm{m}}(x, y, 0)=\boldsymbol{\eta}_{\mathrm{m}}^{\text {init }}(x)$. Herein:

$$
\alpha_{\mathrm{m}}\left(\boldsymbol{\eta}_{\mathrm{m}}\right)=\frac{\lambda_{\mathrm{m}, w} \lambda_{\mathrm{m}, o}}{\lambda_{\mathrm{m}, w}+\lambda_{\mathrm{m}, o}}\left|P_{\mathrm{m}, c}^{\prime}\left(\boldsymbol{\eta}_{\mathrm{m}}\right)\right| .
$$

The properties of the phase mobility should ensure the positive definition of the capillary diffusion parameter $\alpha_{\mathrm{m}}$.

Remark 1. The model (15)-(17) is not completely homogenized. Indeed, the macroscopic equations (15) contain the microscopic function $s_{\mathrm{m}}(x, y, t)$, which can be determined only after solving the cell problem (17). On the other hand, the cell problem (17) contains the macroscopic function $S_{\mathrm{f}}(x, t)$ through the boundary condition, which cannot be eliminated. This means that this problem must be solved for all the cells.

Remark 2. In the case of the local equilibrium in the blocks we have $\boldsymbol{\eta}_{\mathrm{m}}=\mathcal{S}_{\mathrm{m}}=s_{\mathrm{m}}$, according to Section 2.2. Then the macroscopic model (15)-(17) takes the form of the double porosity model for two-phase flow obtained by many authors; see, for example, $[11,24,35,36]$.

Remark 3. In the homogenized model (15) the source term $Q_{w}$ depends on the local saturation in blocks $s_{\mathrm{m}}$, which is the nonlocal functional of Barenblatt's saturation $\boldsymbol{\eta}_{\mathrm{m}}$. Moreover, $\boldsymbol{\eta}_{\mathrm{m}}$ is the solution of the nonstationary cell problem (17); thus, $\boldsymbol{\eta}_{\mathrm{m}}$, in turn, represents a nonlocal functional. Consequently, the source term $\mathbb{Q}_{w}$ in (15) has a double nonlocality.

3.4. Doubly Nonequilibrium Relationship for the Macroscopic Capillary Pressure. Although the macroscopic model is incompletely homogenized, it enables obtaining a general nonequilibrium relationship for the macroscopic capillary pressure, which is sufficient in practice to analyze qualitatively the overall process. It has the following form:

$$
\left\langle P_{\mathrm{m}, c}\left(\boldsymbol{\eta}_{\mathrm{m}}\right)\right\rangle_{\mathrm{m}}-P_{\mathrm{f}, \mathrm{c}}\left(S_{\mathrm{f}}\right)=-\left\langle\phi_{\mathrm{m}} \zeta \frac{\partial \mathcal{S}_{\mathrm{m}}\left[\boldsymbol{\eta}_{\mathrm{m}}\right]}{\partial t}\right\rangle_{\mathrm{m}},
$$

where the function $\zeta=\zeta(x, y, t)$ is the solution of the following cell problem:

$$
\begin{aligned}
\operatorname{div}_{y}\left(K_{\mathrm{m}} \Lambda_{\mathrm{m}}\left(\boldsymbol{\eta}_{\mathrm{m}}\right) \nabla_{y} \zeta\right) & =1 & & \text { in } Y_{\mathrm{m}} \\
\zeta & =0 & & \text { on } \Gamma_{\mathrm{fm}},
\end{aligned}
$$

where

$$
\Lambda_{\mathrm{m}}\left(\boldsymbol{\eta}_{\mathrm{m}}\right)=\frac{\lambda_{\mathrm{m}, w} \lambda_{\mathrm{m}, o}}{\lambda_{\mathrm{m}, w}+\lambda_{\mathrm{m}, o}} .
$$

To prove (19), let us multiply (17) by $\zeta \vartheta$ where $\vartheta(x, t)$ is a nonzero infinitely differentiable function with a compact support in the cylinder $\Omega_{T}$. Integrating over $Y_{\mathrm{m}} \times \Omega_{T}$ we obtain the following relationship:

$$
\begin{aligned}
& \int_{Y_{\mathrm{m}} \times \Omega_{T}} \zeta \phi_{\mathrm{m}} \frac{\partial}{\partial t} \mathcal{S}_{\mathrm{m}}\left[\boldsymbol{\eta}_{\mathrm{m}}\right] \vartheta(x, t) d x d y d t \\
& -\int_{Y_{\mathrm{m}} \times \Omega_{T}} K_{\mathrm{m}} \Lambda_{\mathrm{m}}\left(\boldsymbol{\eta}_{\mathrm{m}}\right) \nabla_{y} \zeta \cdot \nabla_{y}\left[P_{\mathrm{m}, c}\left(\boldsymbol{\eta}_{\mathrm{m}}\right)-P_{\mathrm{f}, c}\left(S_{\mathrm{f}}\right)\right] \\
& \quad \cdot \vartheta(x, t) d x d y d t=0 .
\end{aligned}
$$

Integrating by parts, using Gauss's theorem, taking into account (20) and the fact that $P_{\mathrm{m}, \mathrm{c}}\left(\boldsymbol{\eta}_{\mathrm{m}}\right)=P_{\mathrm{f}, \mathrm{c}}\left(S_{\mathrm{f}}\right)$ on $\Gamma_{\mathrm{fm}}$, we obtain

$$
\begin{aligned}
& \phi_{\mathrm{m}} \int_{Y_{\mathrm{m}} \times \Omega_{T}} \zeta \frac{\partial}{\partial t} \mathcal{S}_{\mathrm{m}}\left[\boldsymbol{\eta}_{\mathrm{m}}\right] \vartheta d x d y d t \\
& \quad+\int_{Y_{\mathrm{m}} \times \Omega_{T}}\left[P_{\mathrm{m}, c}\left(\boldsymbol{\eta}_{\mathrm{m}}\right)-P_{\mathrm{f}, \mathrm{c}}\left(S_{\mathrm{f}}\right)\right] \vartheta d x d y d t=0
\end{aligned}
$$

or

$$
\begin{aligned}
\phi_{\mathrm{m}} \int_{\Omega_{T}} & \left\langle\zeta \frac{\partial}{\partial t} \mathcal{S}_{\mathrm{m}}\left[\boldsymbol{\eta}_{\mathrm{m}}\right]\right\rangle_{\mathrm{m}} \vartheta d x d t \\
& +\int_{\Omega_{T}}\left[\left\langle P_{\mathrm{m}, c}\left(\boldsymbol{\eta}_{\mathrm{m}}\right)\right\rangle_{\mathrm{m}}-P_{\mathrm{f}, \mathrm{c}}\left(S_{\mathrm{f}}\right)\right] \vartheta d x d t=0,
\end{aligned}
$$

which is equivalent to (19).

Note that (19) generalizes the nonequilibrium capillary relationship obtained earlier in [9] for the case of local equilibrium.

\section{Completely Homogenized Model I: Linearized Flow in Blocks}

A linearization of the cell problem (17) is a way to obtain an explicit analytical relationship for the function $\boldsymbol{\eta}_{\mathrm{m}}$, then for $s_{\mathrm{m}}$, and then for the source term $Q_{w}$ in (15) and finally to obtain a completely homogenized model.

4.1. Linearized Nonequilibrium Cell Problem in Blocks. The idea of the linearization and, thus, simplification of the local problem comes from [8]. This approach already enabled obtaining a completely homogenized two-phase flow model in double porosity media in $[9,24]$. In this paper we make use of the constant linearization; that is, we set

$$
\alpha_{\mathrm{m}}\left(\boldsymbol{\eta}_{\mathrm{m}}\right) \approx \widehat{\alpha}_{\mathrm{m}}=\int_{0}^{1} \alpha_{\mathrm{m}}(\varsigma) d \varsigma=\mathrm{const},
$$

where the function $\alpha_{\mathrm{m}}$ is defined in (18). The linearized imbibition equation (17) reads

$$
\begin{aligned}
\phi_{\mathrm{m}} \frac{\partial}{\partial t} \mathcal{S}_{\mathrm{m}}\left[\boldsymbol{\eta}_{\mathrm{m}}\right]-K_{\mathrm{m}} \widehat{\alpha}_{\mathrm{m}} \Delta_{y} \boldsymbol{\eta}_{\mathrm{m}}=0 \quad \text { in } Y_{\mathrm{m}} \times \Omega_{T} ; \\
\boldsymbol{\eta}_{\mathrm{m}}(x, y, t)=\mathscr{P}\left(S_{\mathrm{f}}(x, t)\right) \\
\text { on } \Gamma_{\mathrm{fm}} \times \Omega_{T} \text { with } \mathscr{P}\left(S_{\mathrm{f}}\right)=\left(P_{\mathrm{m}, c}^{-1} \circ P_{\mathrm{f}, c}\right)\left(S_{\mathrm{f}}\right) ; \\
\boldsymbol{\eta}_{\mathrm{m}}(x, y, 0)=\boldsymbol{\eta}_{\mathrm{m}}^{\text {init }}(x) \quad \text { in } Y_{\mathrm{m}} \times \Omega,
\end{aligned}
$$

where we keep the same notation $\boldsymbol{\eta}_{\mathrm{m}}$ as in (17) for the function satisfying the linearized problem (26).

4.2. Separation of the Fast and Slow Variables in the Linearized Cell Problem in Blocks. As mentioned in Remark 1, the main problem of the macroscopic model (15)-(17) consists of the fact that the slow and fast variables are not completely separated. Such a separation can be reached in the considered case of the linearized problem in blocks (26). Indeed, its solution 
can be presented in the following form with separated fast variable $y$ and slow variable $x$ :

$$
\begin{aligned}
\boldsymbol{\eta}_{\mathrm{m}}=\mathrm{w}(y, t) \star \mathscr{P}_{t}^{\prime}(x, t)+ & S_{\mathrm{m}}^{\text {init }}(x) \\
& \text { with } \mathscr{P}_{t}^{\prime}=\frac{\partial \mathscr{P}\left(S_{\mathrm{f}}(x, t)\right)}{\partial t},
\end{aligned}
$$

where the function $\mathrm{w}=\mathrm{w}(y, t)$ is independent of the slow variable $x$ and is the solution of the following auxiliary cell problem:

$$
\begin{aligned}
& \frac{\phi_{\mathrm{m}}}{\tau_{\mathrm{m}}} \frac{\partial}{\partial t}\left(e^{-t / \tau_{\mathrm{m}}} \star \mathrm{w}\right)-K_{\mathrm{m}} \widehat{\alpha}_{\mathrm{m}} \Delta_{y} \mathrm{w}=0 \quad \text { in } Y_{\mathrm{m}} ; \\
& \mathrm{w}(y, t)=1 \quad \text { on } \Gamma_{\mathrm{fm}} ; \\
& \mathrm{w}(y, 0)=0 \quad \text { in } Y_{\mathrm{m}} \text {. }
\end{aligned}
$$

In order to prove (27), we proceed as follows. We will plug the nonequilibrium parameter $\boldsymbol{\eta}_{\mathrm{m}}$ given by (27) in (26) and show that (26) is satisfied. First, we note that it follows from (16) that

$$
\begin{aligned}
\mathcal{S}_{\mathrm{m}}\left[\boldsymbol{\eta}_{\mathrm{m}}\right]= & S_{\mathrm{m}}^{\text {init }} e^{-t / \tau_{\mathrm{m}}} \\
& +\frac{1}{\tau_{\mathrm{m}}}\left[\mathrm{W} \star \mathscr{P}_{t}^{\prime} \star e^{-t / \tau_{\mathrm{m}}}+S_{\mathrm{m}}^{\text {init }} \star e^{-t / \tau_{\mathrm{m}}}\right] \\
= & \frac{1}{\tau_{\mathrm{m}}} \mathrm{W} \star \mathscr{P}_{t}^{\prime} \star e^{-t / \tau_{\mathrm{m}}}+S_{\mathrm{m}}^{\text {init }} .
\end{aligned}
$$

Then

$$
\begin{aligned}
\frac{\partial \mathcal{S}_{\mathrm{m}}\left[\boldsymbol{\eta}_{\mathrm{m}}\right]}{\partial t} & =\frac{1}{\tau_{\mathrm{m}}} \frac{\partial}{\partial t}\left(\mathrm{w} \star \mathscr{P}_{t}^{\prime} \star e^{-t / \tau_{\mathrm{m}}}\right) \\
& =\frac{1}{\tau_{\mathrm{m}}}\left(\mathrm{w} \star \mathscr{P}_{t}^{\prime}-\frac{1}{\tau_{\mathrm{m}}} \mathrm{w} \star \mathscr{P}_{t}^{\prime} \star e^{-t / \tau_{\mathrm{m}}}\right)
\end{aligned}
$$

and from (26), we get

$$
\begin{aligned}
& \frac{\phi_{\mathrm{m}}}{\tau_{\mathrm{m}}}\left(\mathrm{w} \star \mathscr{P}_{t}^{\prime}-\frac{1}{\tau_{\mathrm{m}}} \mathrm{w} \star \mathscr{P}_{t}^{\prime} \star e^{-t / \tau_{\mathrm{m}}}\right) \\
& \quad=K_{\mathrm{m}} \widehat{\alpha}_{\mathrm{m}} \Delta_{y}\left(\mathrm{w} \star \mathscr{P}_{t}^{\prime}\right) \quad \text { in } Y_{\mathrm{m}} \times \Omega_{T} ; \\
& \mathrm{w} \star \mathscr{P}_{t}^{\prime}+S_{\mathrm{m}}^{\text {init }}=\mathscr{P} \quad \text { on } \Gamma_{\mathrm{fm}} \times \Omega_{T} ; \\
& \mathrm{w}(y, 0) \star \mathscr{P}_{t}^{\prime}(x, y, 0)+S_{\mathrm{m}}^{\text {init }}=\eta_{\mathrm{m}}^{\text {init }}(x) .
\end{aligned}
$$

Now, taking into account the definition of the function $\mathrm{w}$ given in (28), the initial conditions $\mathscr{P}(x, 0)=S_{\mathrm{m}}^{\text {init }}$, $\boldsymbol{\eta}_{\mathrm{m}}(x, y, 0)=S_{\mathrm{m}}^{\text {init }}$, and the relations

$$
\begin{aligned}
\mathrm{w}-\frac{1}{\tau_{\mathrm{m}}} \mathrm{W} \star e^{-t / \tau_{\mathrm{m}}} & =\frac{\partial}{\partial t}\left(\mathrm{w} \star e^{-t / \tau_{\mathrm{m}}}\right) ; \\
1 \star \mathscr{P}_{t}^{\prime} & =\int_{0}^{t} \mathscr{P}_{\theta}^{\prime} d \theta=\mathscr{P}(x, t)-\mathscr{P}(x, 0) \\
& =\mathscr{P}(x, t)-S_{\mathrm{m}}^{\text {init }},
\end{aligned}
$$

we observe that the nonequilibrium parameter $\boldsymbol{\eta}_{\mathrm{m}}$ given by (27) is the solution of the boundary value problem (26). This completes the proof of the representation (27).
4.3. Completely Homogenized Model I. Using the obtained solution for the cell problems (27) and (28), as well as the relationship (30), we obtain immediately for the exchange term $\mathbb{Q}_{w}$ in the macroscopic model (15)

$$
\widehat{Q}_{w}=-\frac{\phi_{\mathrm{m}}\left|Y_{\mathrm{m}}\right|}{\tau_{\mathrm{m}}} \frac{\partial}{\partial t}\left(\langle\mathrm{w}\rangle_{\mathrm{m}} \star \frac{\partial \mathscr{P}\left(S_{\mathrm{f}}(x, t)\right)}{\partial t} \star e^{-t / \tau_{\mathrm{m}}}\right),
$$

where the auxiliary function $\mathrm{w}(y, t)$ is the solution of the local problem (28).

It is sufficient to substitute (28) into (15) to obtain (33). Then the macroscopic model (15) takes the form

$$
\begin{aligned}
\phi^{\star} \frac{\partial S_{\mathrm{f}}}{\partial t}-\operatorname{div}_{x}\left\{\mathbb{K} \mathbb{K}^{\star}(x) \lambda_{\mathrm{f}, w}\left(S_{\mathrm{f}}\right) \nabla P_{w}\right\} & =Q_{w}, \\
-\phi^{\star} \frac{\partial S_{\mathrm{f}}}{\partial t}-\operatorname{div}_{x}\left\{\mathbb{K}^{\star}(x) \lambda_{\mathrm{f}, o}\left(S_{\mathrm{f}}\right) \nabla P_{o}\right\} & =-Q_{w}, \\
P_{c}\left(S_{\mathrm{f}}\right) & =P_{o}-P_{w}
\end{aligned}
$$

with the initial condition $S_{\mathrm{f}}(x, 0)=S_{\mathrm{f}}^{\text {init }}(x)$.

The local saturation in blocks $s_{\mathrm{m}}(x, y, t)$ is calculated as

$$
\begin{aligned}
s_{\mathrm{m}}(x, y, t)= & \frac{1}{\tau_{\mathrm{m}}} \mathrm{w}(y, t) \star \frac{\partial \mathscr{P}\left(S_{\mathrm{f}}(x, t)\right)}{\partial t} \star e^{-t / \tau_{\mathrm{m}}} \\
& +S_{\mathrm{m}}^{\text {init }} .
\end{aligned}
$$

The averaged saturation in blocks $\left\langle s_{\mathrm{m}}\right\rangle_{\mathrm{m}}$ and the averaged Barenblatt pseudo-saturation $\left\langle\boldsymbol{\eta}_{\mathrm{m}}\right\rangle_{\mathrm{m}}$ are calculated as

$$
\begin{aligned}
\left\langle s_{\mathrm{m}}\right\rangle_{\mathrm{m}}= & \frac{1}{\tau_{\mathrm{m}}}\langle\mathrm{w}\rangle_{\mathrm{m}} \star \frac{\partial \mathscr{P}\left(S_{\mathrm{f}}(x, t)\right)}{\partial t} \star e^{-t / \tau_{\mathrm{m}}} \\
& +S_{\mathrm{m}}^{\text {init }}(x), \\
\left\langle\boldsymbol{\eta}_{\mathrm{m}}\right\rangle_{\mathrm{m}}= & \langle\mathrm{w}\rangle_{\mathrm{m}} \star \frac{\partial \mathscr{P}\left(S_{\mathrm{f}}(x, t)\right)}{\partial t}+S_{\mathrm{m}}^{\text {init }}(x) .
\end{aligned}
$$

The averaged capillary pressure in the blocks can be calculated directly as $\left\langle P_{\mathrm{m}, c}\left(S_{\mathrm{m}}\right)\right\rangle_{\mathrm{m}}$ since the function $P_{\mathrm{m}, c}(s)$ is given.

The general relationship between the capillary pressures (19) becomes then useless. In addition, it remains noncompletely homogenized, since the function $\zeta(x, y, t)$ defined by problem (20) remains dependent on $\boldsymbol{\eta}_{\mathrm{m}}$ and so forth.

Remark 4. (1) Although the problem is completely homogenized, the calculation of the averaged functions and coefficients requires solving the cell problem (28) for the integrodifferential equation.

(2) The double convolution integral in the macroscopic model (34) clearly shows the appearance of the double memory phenomenon, one of which is caused by the blockfracture interaction while the second one is produced by the microscale nonequilibrium in blocks.

\section{Completely Homogenized Model II: Low Contrast in Block-Fracture Properties}

The second way to obtain a completely homogenized model is to assume that the block permeability $\varepsilon^{2} K_{\mathrm{m}}$ is still very low 
with respect to fractures, but the contrast in the permeability is not so high as previously. Then the parameter $K_{m}$ in (3) should be sufficiently high. Its order, however, should not disturb the asymptotic expansions (12), which is ensured if

$$
\varepsilon^{2} K_{\mathrm{m}} \sim \frac{\varepsilon^{2}}{\varkappa}, \quad \varepsilon \ll \varkappa \ll 1
$$

The fact that the contrast in block-fracture properties is not very high means that the delay in the block behavior is expected to be not very high. Thus we deal with the case of a low macroscopic nonequilibrium.

The macroscopic model in this case may be obtained from the general model (15), (16), (17) by asymptotic expansion with respect to parameter $\varkappa$.

The justification to the fact that the expansion over two small parameters $\varepsilon, \varkappa$ is commutative may be done in the following way. According to [7], the block's contribution to the macroscopic model is double: (i) they form a source flow from the block center towards fracture, which determines the exchange terms $Q$ in (15); and (ii) they can contribute to the effective permeability if the block permeability is not too low. For the case of the block permeability equal to $\varepsilon^{2} K_{m}$ with $K_{\mathrm{m}} \sim 1$, the macroscopic model (15) contains only the source term but does not contain the block contribution to the effective permeability (ctep). If we progressively increase the block permeability, then the source flow will decrease, but ctep will grow. The source flow and ctep become of the same order when $K_{\mathrm{m}} \sim \varepsilon^{-1}$. If $1 \ll K_{\mathrm{m}} \ll \varepsilon^{-1}($ or $\varepsilon \ll \varkappa \ll 1$ ), the source flow is more important than ctep. In this case, the ctep may be still neglected; then the macroscopic model is analogous to (15), but the source terms will have smaller order and may be expand asymptotically over $\varkappa$. This corresponds exactly to the case (37).

5.1. Asymptotic Expansions with respect to $\varkappa$. All functions in the general homogenized model (15), (16), (17) may be presented in the form

$$
\begin{aligned}
S_{\mathrm{f}}(x, t) & =\widehat{S}_{\mathrm{f}}(x, t)+\varkappa S_{\mathrm{f}, 1}(x, t)+\mathcal{O}\left(\varkappa^{2}\right), \\
s_{\mathrm{m}}(x, y, t) & =\widehat{S}_{\mathrm{m}}(x, t)+\varkappa S_{\mathrm{m}, 1}(x, y, t)+\mathcal{O}\left(\varkappa^{2}\right), \\
P_{\mathrm{f}, w}(x, t) & =\widehat{P}_{\mathrm{f}, w}(x, t)+\mathcal{O}(\varkappa), \\
P_{\mathrm{f}, o}(x, t) & =\widehat{P}_{\mathrm{f}, o}(x, t)+\mathcal{O}(\varkappa), \\
\eta_{\mathrm{m}}(x, y, t) & =\widehat{\boldsymbol{\eta}}_{\mathrm{m}}(x, t)+\varkappa \eta_{\mathrm{m}, 1}(x, y, t)+\mathcal{O}\left(\varkappa^{2}\right), \\
\zeta(y, x, t) & =\varkappa \zeta_{1}(x, y, \mathrm{t})+\mathcal{O}\left(\varkappa^{2}\right),
\end{aligned}
$$

where $\widehat{S}_{\mathrm{f}}, \widehat{S}_{\mathrm{m}}$, and so forth are the average values over the fractures and the block, respectively. Consequently to such a structure of the expansion, the functions of the first approximation satisfy the following property:

$$
\left\langle S_{\mathrm{f}, 1}\right\rangle_{\mathrm{f}}=\left\langle S_{\mathrm{m}, 1}\right\rangle_{\mathrm{m}}=\left\langle\boldsymbol{\eta}_{\mathrm{m}, 1}\right\rangle_{\mathrm{m}}=0 .
$$

According to [11], in this case the first term $\widehat{S}_{\mathrm{m}}(x, t)$ does not depend on the fast variable $y$, differently from the case which is justified by the low contrast in the medium heterogeneity and, consequently, by the sufficiently fast stabilization of the saturation field in blocks.

5.2. Doubly Nonequilibrium Capillary Pressure. In this subsection we obtain an explicit, completely homogenized relationship for the nonequilibrium capillary pressure. It reads

$$
\begin{aligned}
& P_{\mathrm{m}, c}\left(\widehat{\boldsymbol{\eta}}_{\mathrm{m}}\right)-P_{\mathrm{f}, \mathrm{c}}\left(\widehat{S}_{\mathrm{f}}\right)=\tau_{\mathrm{macr}}\left(\widehat{\boldsymbol{\eta}}_{\mathrm{m}}\right) \frac{\partial \widehat{S}_{\mathrm{m}}}{\partial t}, \\
& \widehat{\boldsymbol{\eta}}_{\mathrm{m}}=\widehat{S}_{\mathrm{m}}+\tau_{\mathrm{m}} \frac{\partial \widehat{S}_{\mathrm{m}}}{\partial t} .
\end{aligned}
$$

Here the macroscopic relaxation time $\tau_{\text {macr }}\left(\widehat{\boldsymbol{\eta}}_{\mathrm{m}}\right)$ is given by

$$
\tau_{\text {macr }}\left(\widehat{\boldsymbol{\eta}}_{\mathrm{m}}\right)=\frac{\varkappa}{\Lambda_{\mathrm{m}}\left(\widehat{\boldsymbol{\eta}}_{\mathrm{m}}\right)}\left\langle\phi_{\mathrm{m}} \boldsymbol{\varphi}\right\rangle_{\mathrm{m}},
$$

where $\Lambda_{\mathrm{m}}$ is defined in (21) and $\varphi(y)$ is the solution of the auxiliary cell problem in $Y_{\mathrm{m}}$ :

$$
\begin{aligned}
\operatorname{div}_{y}\left(K_{\mathrm{m}} \nabla_{y} \varphi\right) & =1, \quad \text { in } Y_{\mathrm{m}} \\
\varphi & =0 \quad \text { on } \Gamma_{\mathrm{fm}}
\end{aligned}
$$

In order to prove (40), we make use of the global relationship for the macroscopic capillary pressure, (19), and the asymptotic expansions given in the beginning of the section. We have

$$
\begin{gathered}
\left\langle P_{\mathrm{m}, c}\left(\widehat{\boldsymbol{\eta}}_{\mathrm{m}}+\varkappa \boldsymbol{\eta}_{\mathrm{m}, 1}\right)\right\rangle_{\mathrm{m}}-P_{\mathrm{f}, \mathrm{c}}\left(\widehat{S}_{\mathrm{f}}+\varkappa S_{\mathrm{f}, 1}\right) \\
=-\varkappa\left\langle\phi_{\mathrm{m}} \boldsymbol{\zeta}_{1}\right\rangle_{\mathrm{m}} \frac{\partial \widehat{S}_{\mathrm{m}}}{\partial t}+\mathcal{O}\left(\varkappa^{2}\right) .
\end{gathered}
$$

Developing the corresponding terms in Taylor's series, from this equation, one easily gets

$$
\begin{aligned}
P_{\mathrm{m}, c}\left(\widehat{\boldsymbol{\eta}}_{\mathrm{m}}\right)+\varkappa P_{\mathrm{m}, c}^{\prime}\left(\widehat{\boldsymbol{\eta}}_{\mathrm{m}}\right)\left\langle\boldsymbol{\eta}_{\mathrm{m}, 1}\right\rangle_{\mathrm{m}}-P_{\mathrm{f}, \mathrm{c}}\left(\widehat{S}_{\mathrm{f}}\right) \\
\quad+\varkappa P_{\mathrm{f}, c}^{\prime}\left(\widehat{S}_{\mathrm{f}}\right) S_{\mathrm{f}, 1}=-\varkappa\left\langle\phi_{\mathrm{m}} \zeta_{1}\right\rangle_{\mathrm{m}} \frac{\partial \widehat{S}_{\mathrm{m}}}{\partial t}+\mathcal{O}\left(\varkappa^{2}\right) .
\end{aligned}
$$

Taking now the average over the fracture part and applying (39), we have

$$
P_{\mathrm{m}, c}\left(\widehat{\boldsymbol{\eta}}_{\mathrm{m}}\right)-P_{\mathrm{f}, \mathrm{c}}\left(\widehat{S}_{\mathrm{f}}\right)=-\varkappa\left\langle\phi_{\mathrm{m}} \zeta_{1}\right\rangle_{\mathrm{m}} \frac{\partial \widehat{S}_{\mathrm{m}}}{\partial t}+\mathcal{O}\left(\varkappa^{2}\right) .
$$

Moreover, it follows from (20) that the function $\zeta_{1}$ satisfies the following equation:

$$
\begin{aligned}
\Lambda_{\mathrm{m}}\left(\widehat{\boldsymbol{\eta}}_{\mathrm{m}}\right) \operatorname{div}_{y}\left(K_{\mathrm{m}} \nabla_{y} \zeta_{1}\right)=1 & \text { in } Y_{\mathrm{m}} \\
\zeta_{1}=0 & \text { on } \Gamma_{\mathrm{fm}} .
\end{aligned}
$$

Setting $\zeta_{1}=\left(1 / \Lambda_{\mathrm{m}}\left(\widehat{\boldsymbol{\eta}}_{\mathrm{m}}\right)\right) \boldsymbol{\varphi}(y)$, we arrive at (42). Substituting the latter relationship in (45), we obtain, finally, (40). 
Remark 5. The expression (40) is a generalization of the nonequilibrium relationship (38c) of Bourgeat and Panfilov obtained in [11], which takes into account the double nonequilibrium: the microscopic phase redistribution in the blocks characterized by the microscopic relaxation time $\tau_{\mathrm{m}}$ and the macroscopic relaxation time $\tau_{\text {macr }}$ characterized by the delay in phase redistribution between the blocks and the fractures. Thus, the low contrast in medium properties, which corresponds to sufficiently weak nonstationary effects within the blocks, leads to the appearance of a short macroscopic memory in the upscaled system, with small relaxation time $\tau_{\text {macr }}$.

5.3. Completely Homogenized Model II. From (15) and (40) we obtain the following completely upscaled model:

$$
\begin{aligned}
& \phi^{\star} \frac{\partial \widehat{S}_{\mathrm{f}}}{\partial t}-\operatorname{div}_{x}\left\{\mathbb{K}^{\star}(x) \lambda_{\mathrm{f}, w}\left(\widehat{S}_{\mathrm{f}}\right) \nabla \widehat{P}_{w}\right\} \\
& \quad=-\phi_{\mathrm{m}}\left|Y_{\mathrm{m}}\right| \frac{\partial \widehat{S}_{\mathrm{m}}}{\partial t}, \\
& -\phi^{\star} \frac{\partial \widehat{S}_{\mathrm{f}}}{\partial t}-\operatorname{div}_{x}\left\{\mathbb{K}^{\star}(x) \lambda_{\mathrm{f}, o}\left(\widehat{S}_{\mathrm{f}}\right) \nabla \widehat{P}_{o}\right\} \\
& \quad=\phi_{\mathrm{m}}\left|Y_{\mathrm{m}}\right| \frac{\partial \widehat{S}_{\mathrm{m}}}{\partial t}, \\
& \widehat{P}_{o}-\widehat{P}_{w}=P_{c}\left(\widehat{S}_{\mathrm{f}}\right), \\
& P_{\mathrm{m}, c}\left(\widehat{\boldsymbol{\eta}}_{\mathrm{m}}\right)-P_{\mathrm{f}, c}\left(\widehat{S}_{\mathrm{f}}\right)=\tau_{\mathrm{macr}}\left(\widehat{\boldsymbol{\eta}}_{\mathrm{m}}\right) \frac{\partial \widehat{S}_{\mathrm{m}}}{\partial t}, \\
& \quad \widehat{\boldsymbol{\eta}}_{\mathrm{m}}=\widehat{S}_{\mathrm{m}}+\tau_{\mathrm{m}} \frac{\partial \widehat{S}_{\mathrm{m}}}{\partial t}
\end{aligned}
$$

with the initial condition $\widehat{S}_{f}(x, 0)=S_{f}^{\text {init }}(x)$.

This system of five equations represents the closed completely homogenized model with respect to five functions $\widehat{S}_{f}$, $\widehat{S}_{\mathrm{m}}, \widehat{\boldsymbol{\eta}}_{\mathrm{m}}, \widehat{P}_{o}$, and $\widehat{P}_{w}$.

Note that the latter relationship for the capillary pressure in (47) represents the equation that defines the saturation in blocks $\widehat{S}_{\mathrm{m}}$ (as the solution of the nonlinear ordinary differential equation of the first order).

5.4. Double Nonequilibrium Capillary Relationship for Small Microscopic Relaxation Time. The relationship (40) can be sufficiently simplified if we assume that Barenblatt's time $\tau_{\mathrm{m}} \sim$ $\varkappa$. Then

$$
\begin{aligned}
& P_{\mathrm{m}, c}\left(\widehat{S}_{\mathrm{m}}\right)-P_{\mathrm{f}, \mathrm{c}}\left(\widehat{S}_{\mathrm{f}}\right) \\
& \quad=\left[\tau_{\text {macr }}\left(\widehat{S}_{\mathrm{m}}\right)+\tau_{\mathrm{m}}\left|\frac{d P_{\mathrm{m}, \mathrm{c}}}{d \widehat{S}_{\mathrm{m}}}\right|\right] \frac{\partial \widehat{S}_{\mathrm{m}}}{\partial t},
\end{aligned}
$$

where $\tau_{\text {macr }}$ is defined through (41).

Then, in the case of low contrast in medium properties and low microscopic disequilibrium, we obtain the kinetic relationship for the capillary pressure in blocks, in which the general relaxation time represents a weighted sum of the microscopic and the macroscopic relaxation times.

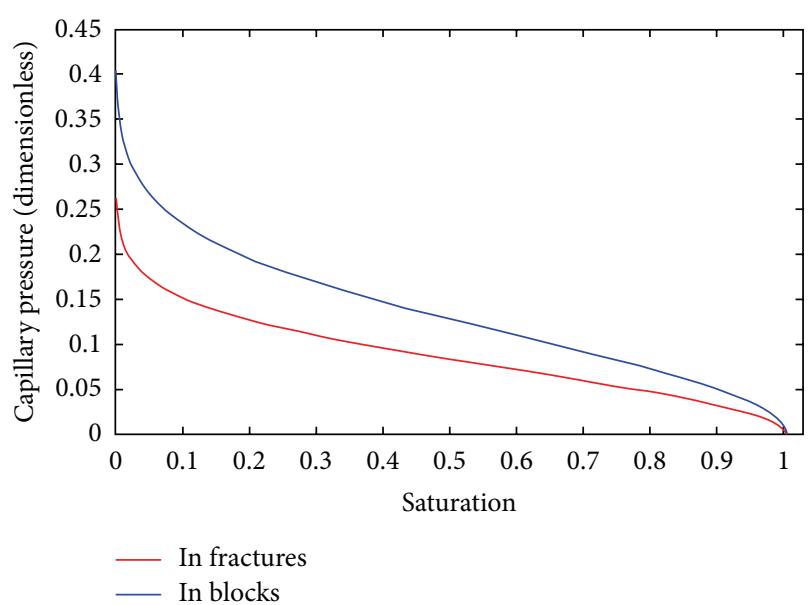

FIGURE 2: Capillary pressure as function of saturation for blocks and fractures.

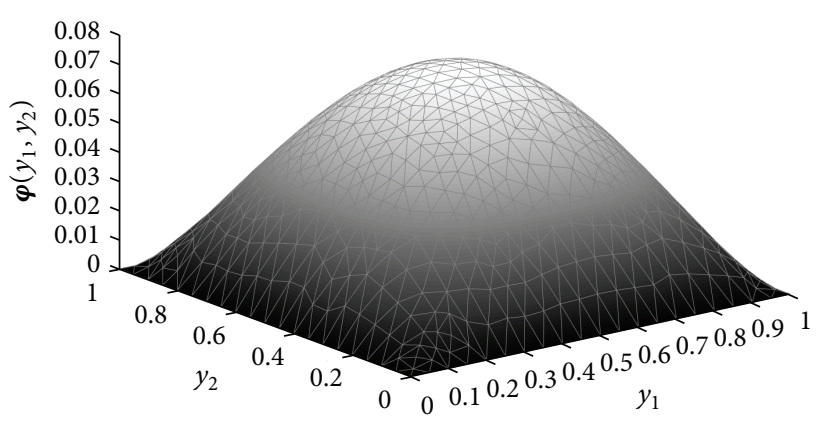

Figure 3: Solution of the cell problem (42).

\section{Analysis of the Impact of the Double Nonequilibrium}

To better understand the impact of the local nonequilibrium on the macroscopic behavior, we consider two test cases based on the completely homogenized model (47).

Let us assume that the saturation in fractures is constant in space and is a given function of time, whose behavior reflects the physical process we are analyzing. The capillary pressures are described by the functions

$$
\begin{gathered}
P_{\mathrm{f}, \mathrm{c}}\left(S_{\mathrm{f}}\right)=\gamma \sqrt{-\ln \left(S_{\mathrm{f}}\right)}, \\
P_{\mathrm{m}, \mathrm{c}}\left(S_{\mathrm{m}}\right)=\frac{\gamma}{\delta} \sqrt{-\ln \left(S_{\mathrm{m}}\right)},
\end{gathered}
$$

where $\gamma=0.1$ and $\delta=0.65$. These functions are shown in Figure 2.

The relative permeability is $k_{r w}(s)=s^{2}, k_{r o}(s)=(1-s)^{2}$ both for blocks and for fractures. Other parameters are $K_{\mathrm{f}}=$ $1, \varepsilon=0.1, \varkappa=0.25$ (i.e., $\left.K_{\mathrm{m}}=4\right), \phi_{\mathrm{m}}=0.2$, the volume fraction of blocks $\Theta=0.9, \mu_{w} / \mu_{o}=0.5$.

The cell problem (42) was solved numerically in a square by a finite element method using the MATLAB PDE Toolbox. The solution and the numerical grid are presented in Figure 3. The average value $\langle\varphi\rangle_{\mathrm{m}} \approx 0.025$. 


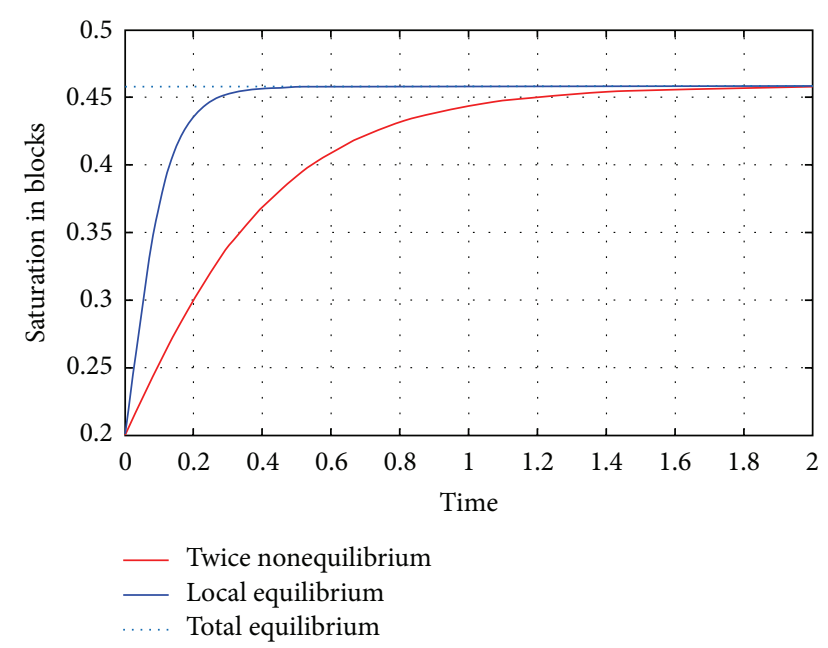

Figure 4: Evolution in time of the saturation in blocks from the initial nonequilibrium state; at constant saturation in fractures.

We analyzed two tests, in which the initial state was in local disequilibrium within blocks and in macroscale disequilibrium between blocks and fractures. We maintained a given behavior of the saturation in fractures and analyzed the answer of the blocks on such a perturbation. The saturation in blocks, $\widehat{S}_{\mathrm{m}}$, was calculated from the differential equation (48):

$$
\begin{aligned}
& {\left[\tau_{\text {macr }}\left(\widehat{S}_{\mathrm{m}}\right)+\tau_{\mathrm{m}}\left|\frac{d P_{\mathrm{m}, c}}{d \widehat{S}_{\mathrm{m}}}\right|\right] \frac{\partial \widehat{S}_{\mathrm{m}}}{\partial t}-P_{\mathrm{m}, c}\left(\widehat{S}_{\mathrm{m}}\right)} \\
& \quad=-P_{\mathrm{f}, c}\left(\widehat{S}_{\mathrm{f}}\right)
\end{aligned}
$$

with the initial condition $\widehat{S}_{\mathrm{m}}(t=0)=S_{\mathrm{m}}^{\text {init }}$.

Test Case 1. In the first test, the saturation in fractures, $S_{f}(t)$, was constant in time and equal to 0.155 . The corresponding equilibrium saturation in blocks was 0.455 ; however the initial saturation in blocks, $S_{\mathrm{m}}^{\text {init }}$, was equal to 0.2 , which was in high disequilibrium.

Such a disequilibrium causes the spontaneous evolution of the saturation in blocks, which tends to the equilibrium value, as shown in Figure 4.

As expected, the time of reaching the total equilibrium is higher in the case of double disequilibrium.

Test Case 2. In the second test, the saturation in fractures, $S_{\mathrm{f}}(t)$, periodically oscillated in time, which corresponds in practice to the functioning of an underground storage of injected gas in an aquifer. In this case, the saturation is that of the injected gas. The increase of the injected saturation means the period of gas injection, while the saturation minimums correspond to gas extraction. If the system was in total equilibrium, then its behavior would be such that is shown in Figure 5.

The true behavior of the saturation in blocks is shown in Figure 6, where the initial saturation in blocks, $S_{\mathrm{m}}^{\text {init }}$, was in the nonequilibrium with fractures.

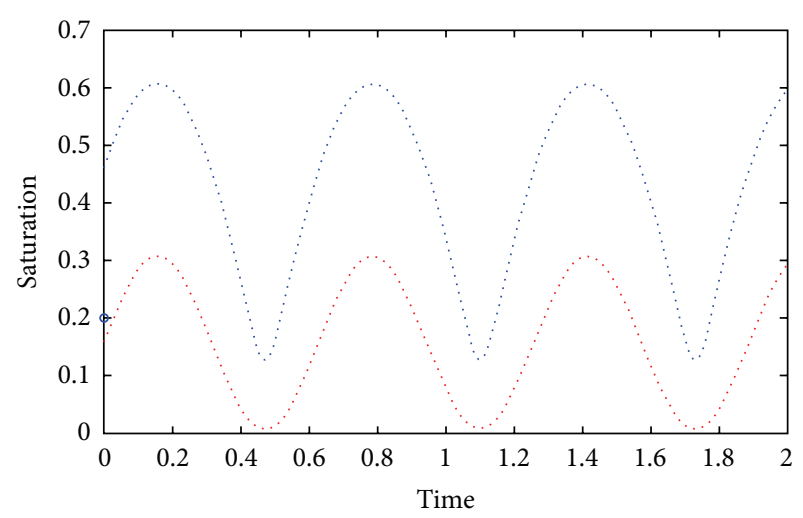

- Initial saturation in blocks

Saturation in blocks in equilibrium with fractures Saturation in fractures in equilibrium with blocks

FIGURE 5: Saturation in blocks and fractures in the case of the total equilibrium between them.

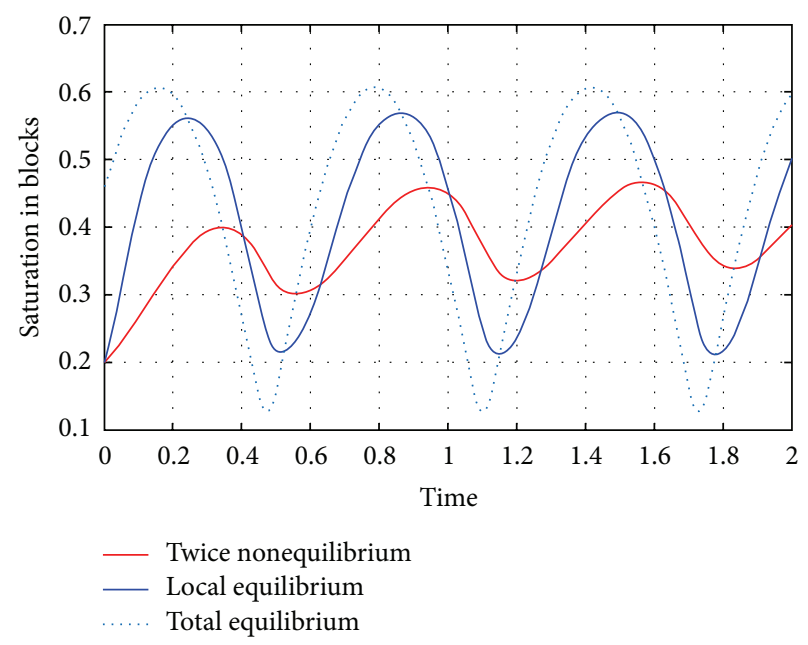

FIGURE 6: Evolution in time of the saturation in blocks from the initial nonequilibrium state; at periodically oscillating saturation in fractures.

As seen, the saturation in blocks is in permanent delay with respect to the equilibrium curve. The delay is expressed in two main forms:

(i) the minimum and the maximum values of saturation are very different from the equilibrium case;

(ii) the phase-shift is observed for the nonequilibrium case.

Both effects are higher for the case of the double nonequilibrium.

Therefore, the local Barenblatt disequilibrium certainly increases the degree of the nonequilibrium in the system but also can lead to very different qualitative behavior of the saturation field, such that the equilibrium behavior can be never reached, as in the example with underground gas storage. 


\section{Conclusion}

We have developed, using homogenization, a new effective model of two-phase flow in double porosity media which takes into account the local disequilibrium of phase redistribution, introduced first by Barenblatt. This process can be very special in low permeable media. The obtained model is more general than those developed previously in $[8,9]$ for the case of the local equilibrium.

In the case of low contrast in block-fracture properties, the model may be completely homogenized and reduced to system (47), in which the last relationship relates the average capillary pressures in blocks and fractures. The total relaxation time is explicitly calculated as a function of the average saturation in blocks. It includes two terms: the microscale time of Barenblatt and the macroscale time of delay between block and fractures. These two components of the relaxation times underline the double nonequilibrium in the system: one of them appears on the microscale in the form of the noninstantaneous phase redistribution, while the second one is the permanent delay between the saturations within the blocks and the fractures.

The simulations we have performed show that the nonequilibrium can have very important impact on the behavior of the fluids, especially in the case of oscillating regimes typical, for instance, for underground gas storages. In this case, the average saturation follows a strict nonequilibrium behavior and can never reach the equilibrium state.

\section{Appendix}

\section{Derivation of the Homogenized Model (15)-(17)}

(1) Substituting the asymptotic expansion (12) in (6)-(8) and grouping the terms of the same order, we obtain the following system of $\varepsilon^{-1}, \varepsilon^{0}$, and $\varepsilon^{1}$ equations needed to obtain the homogenized model (in all the equations below $j=w, o$ ):

(i) for $y \in Y_{\mathrm{f}}$ :

$$
\begin{aligned}
& \sim \mathcal{E}^{-1}:-\operatorname{div}_{y}\left\{\lambda_{\mathrm{f}, j}\left(S_{\mathrm{f}}\right)\left(\nabla_{x} P_{\mathrm{f}, j}+\nabla_{y} p_{\mathrm{f}, j}^{(1)}\right)\right\}=0 \\
& \sim \varepsilon^{0}:\left\{\begin{array}{l} 
\pm \phi_{\mathrm{f}} \frac{\partial S_{\mathrm{f}}}{\partial t}-\operatorname{div}_{x}\left\{K_{\mathrm{f}} \lambda_{\mathrm{f}, j}\left(S_{\mathrm{f}}\right)\left(\nabla_{x} P_{\mathrm{f}, j}+\nabla_{y} p_{\mathrm{f}, j}^{(1)}\right)\right\}-\operatorname{div}_{y}\left\{Z_{\mathrm{f}, j}\right\}=0, \\
P_{\mathrm{f}, o}-P_{\mathrm{f}, w}=P_{\mathrm{f}, c}\left(S_{\mathrm{f}}\right),
\end{array}\right.
\end{aligned}
$$

where “+” is for $j=w$, “-” is for $j=o$, and

$$
\begin{aligned}
& \mathrm{Z}_{\mathrm{f}, j} \stackrel{\text { def }}{=} K_{\mathrm{f}}\left[\lambda_{\mathrm{f}, j}\left(S_{\mathrm{f}}\right)\left(\nabla_{x} p_{\mathrm{f}, j}^{(1)}+\nabla_{y} p_{\mathrm{f}, j}^{(2)}\right)\right. \\
&\left.+\lambda_{\mathrm{f}, j}^{\prime}\left(S_{\mathrm{f}}\right) S_{\mathrm{f}}^{(1)}\left(\nabla_{x} P_{\mathrm{f}, j}+\nabla_{y} p_{\mathrm{f}, j}^{(1)}\right)\right]
\end{aligned}
$$

(ii) for $y \in Y_{\mathrm{m}}$ :

$$
\begin{aligned}
& \sim \varepsilon^{0}: \\
& \qquad\left\{\begin{array}{l} 
\pm \phi_{\mathrm{m}} \frac{\partial s_{\mathrm{m}}}{\partial t}-\operatorname{div}_{y}\left\{K_{\mathrm{m}} \lambda_{\mathrm{m}, j}\left(\boldsymbol{\eta}_{\mathrm{m}}\right) \nabla_{y} p_{\mathrm{m}, j}\right\}=0, \\
p_{\mathrm{m}, o}-p_{\mathrm{m}, w}=P_{\mathrm{m}, c}\left(\boldsymbol{\eta}_{\mathrm{m}}\right) ;
\end{array}\right.
\end{aligned}
$$

(iii) for $y \in \Gamma_{\mathrm{fm}}$ :

$$
\begin{aligned}
& \sim \varepsilon^{0}:\left\{\begin{array}{l}
K_{\mathrm{f}} \lambda_{\mathrm{f}, j}\left(S_{\mathrm{f}}\right)\left(\nabla_{x} P_{\mathrm{f}, j}+\nabla_{y} p_{\mathrm{f}, j}^{(1)}\right) \cdot \vec{v}=0, \\
p_{\mathrm{m}, j}=P_{\mathrm{f}, j},
\end{array}\right. \\
& \sim \varepsilon^{1}: \mathrm{Z}_{\mathrm{f}, j} \cdot \vec{v}=K_{\mathrm{m}} \lambda_{\mathrm{m}, j}\left(\boldsymbol{\eta}_{\mathrm{m}}\right) \nabla_{y} p_{\mathrm{m}, j} \cdot \vec{v},
\end{aligned}
$$

where $\vec{\nu}$ is normal to $\Gamma_{\mathrm{fm}}$ and is directed from $Y_{\mathrm{f}}$ to $Y_{\mathrm{m}}$.
(2) Integrating each equation (A.2) over the fractures $Y_{\mathrm{f}}$ and taking into account that, for any $Y$-periodic vector $\mathbf{f}: \int_{Y_{\mathrm{f}}} \operatorname{div}_{y} \mathbf{f} d y=\int_{\Gamma_{\mathrm{fm}}} \mathbf{f} \cdot \vec{v} d y$, we obtain

$$
\begin{aligned}
& \pm \phi_{\mathrm{f}}\left|Y_{\mathrm{f}}\right| \frac{\partial S_{\mathrm{f}}}{\partial t} \\
& \quad-\int_{Y_{\mathrm{f}}} \operatorname{div}_{x}\left\{K_{\mathrm{f}} \lambda_{\mathrm{f}, j}\left(S_{\mathrm{f}}\right)\left(\nabla_{x} P_{\mathrm{f}, j}+\nabla_{y} p_{f, j}^{(1)}\right)\right\} d y \\
& \quad=Q_{j},
\end{aligned}
$$

where $\mathbb{Q}_{j}=\int_{\Gamma_{\mathrm{fm}}} \mathrm{Z}_{\mathrm{f}, j} \cdot \vec{v} d y$, or, using (A.6), $\mathbb{Q}_{j}=$ $\int_{\Gamma_{\mathrm{fm}}} K_{\mathrm{m}} \lambda_{\mathrm{m}, j}\left(\boldsymbol{\eta}_{\mathrm{m}}\right) \nabla_{y} p_{\mathrm{m}, j} \cdot \vec{\nu} d y$.

Using the theorem of Gauss and the first equation (A.4):

$$
\begin{aligned}
\mathbb{Q}_{j} & =-\int_{Y_{\mathrm{m}}} \operatorname{div}_{y}\left(K_{\mathrm{m}} \lambda_{\mathrm{m}, j}\left(\boldsymbol{\eta}_{\mathrm{m}}\right) \nabla_{y} p_{\mathrm{m}, j}\right) d y \\
& =\int_{Y_{\mathrm{m}}} \mp \phi_{\mathrm{m}} \frac{\partial s_{\mathrm{m}}}{\partial t} d y=\mp \phi_{\mathrm{m}}\left|Y_{\mathrm{m}}\right| \frac{\partial\left\langle s_{\mathrm{m}}\right\rangle_{\mathrm{m}}}{\partial t} .
\end{aligned}
$$

(3) Equation (A.1) enables separating the slow $(x)$ and the fast $(y)$ variables in the form of a series:

$$
p_{\mathrm{f}, j}^{(1)}(x, y, t)=\sum_{k=1}^{d} \zeta_{k}(y) \frac{\partial P_{\mathrm{f}, j}}{\partial x_{k}}(x, t)+C_{j}(x, t),
$$


where the functions $C_{j}$ are not defined but will disappear from the average model, while the functions $\zeta_{k}(k=1, \ldots, d)$ are the solutions to the cell problems (14) resulting from (A.1).

(4) Substituting (A.9) into (A.7) we obtain the final form of the averaged equations:

$$
\pm \phi_{\mathrm{f}}\left|Y_{\mathrm{f}}\right| \frac{\partial S_{\mathrm{f}}}{\partial t}-\operatorname{div}_{x}\left\{\mathbb{K}^{\star} \lambda_{\mathrm{f}, j}\left(S_{\mathrm{f}}\right) \nabla_{x} P_{\mathrm{f}, j}\right\}=Q_{j}
$$

which is the macroscopic model (15). Herein $\mathbb{K}^{\star}$ is defined in Section 3.2.

(5) The local problem (A.4) can be reduced to the equivalent form (17).

Let us introduce the total Darcy velocity:

$$
\begin{aligned}
V & \stackrel{\text { def }}{=}-K_{\mathrm{m}}\left[\lambda_{\mathrm{m}, w} \nabla_{y} p_{\mathrm{m}, w}+\lambda_{\mathrm{m}, o} \nabla_{y} p_{\mathrm{m}, o}\right] \\
& =-K_{\mathrm{m}}\left[\lambda_{\mathrm{m}} \nabla_{y} p_{\mathrm{m}, w}+\lambda_{\mathrm{m}, o} \nabla_{y} P_{\mathrm{m}, c}\right]
\end{aligned}
$$

where $\lambda_{\mathrm{m}}=\lambda_{\mathrm{m}, w}+\lambda_{\mathrm{m}, \mathrm{o}}$. yields

Then the sum of two equations (A.4) for $j=w$ and $j=o$

$$
\nabla_{y} \cdot V=0, \quad y \in Y_{\mathrm{m}} .
$$

Along the interface $\Gamma_{\mathrm{fm}}$ the tangent velocity $V_{\tau}$ is zero, since the pressures $p_{\mathrm{m}, w}$ and $p_{\mathrm{m}, o}$ are independent of $y$, according to the second boundary condition in (A.5). Then (A.12) with the boundary condition $\left.V_{\tau}\right|_{\Gamma_{\mathrm{fm}}}=0$ has the trivial solution: $V=0, y \in Y_{\mathrm{m}}$.

Let us introduce the fractional flow of water: $F \stackrel{\text { def }}{=}$ $\lambda_{\mathrm{m}, w} / \lambda_{\mathrm{m}}$. Then

$$
\begin{aligned}
K_{\mathrm{m}} \lambda_{\mathrm{m}, w} \nabla_{y} p_{\mathrm{m}, w} & =-F\left(V+K_{\mathrm{m}} \lambda_{\mathrm{m}, o} \nabla_{y} P_{\mathrm{m}, c}\right) \\
& =-K_{\mathrm{m}} \lambda_{\mathrm{m}, o} \nabla_{y} P_{\mathrm{m}, c} .
\end{aligned}
$$

Then from the first equation (A.4) we obtain

$$
\phi_{\mathrm{m}} \frac{\partial s_{\mathrm{m}}}{\partial t}=-\nabla_{y} \cdot\left(K_{\mathrm{m}} \lambda_{\mathrm{m}, o} F \nabla_{y} P_{\mathrm{m}, c}\right)
$$

which is (17).

\section{Competing Interests}

The authors declare that they have no competing interests.

\section{Acknowledgments}

The work of B. Amaziane and L. Pankratov was partially supported by the Carnot Institute, ISIFoR Project (Institute for the Sustainable Engineering of Fossil Resources), whose support is gratefully acknowledged.

\section{References}

[1] G. I. Barenblatt, "Flow of two immiscible liquids through the homogeneous porous medium," Izvestiya Academii Nauk SSSR, Seriya Mekhanika Zhidkosti i Gaza, vol. 5, pp. 144-151, 1971.
[2] A. P. Vinichenko, "Theory of non stationary non-equilibrium flow of immiscible fluids," in Izvestiia Akademii Nauk SSSR, vol. 9 of Mekhanika Zhidkosti i Gaza, pp. 51-58, 1978.

[3] G. I. Barenblatt and A. P. Vinichenko, "Non-equilibrium flow of immiscible fluids in porous media," Uspekhi Matematicheskikh Nauk, vol. 3, pp. 35-50, 1980.

[4] V. N. Nikolaevskii, E. A. Bondarev, M. I. Mirkin, G. Stepanova, and V. Terzi, Transport of Hydrocarbure Mixtures in Porous Media, Nedra, Moscow, Russia, 1968 (Russian).

[5] C. M. Marle, "From the pore scale to the macroscale: equations governing multiphase fluid flow through porous media," in Proceedings of the Euromech 143, A. Verruijt and F. D. J. Barends, Eds., Balkema, September 1981.

[6] S. M. Hassanizadeh and W. G. Gray, "Thermodynamic basis of capillary pressure in porous media," Water Resources Research, vol. 29, no. 10, pp. 3389-3405, 1993.

[7] M. Panfilov, Macroscale Models of Flow through Highly Heterogeneous Porous Media, Kluwer Academic Publishers, London, UK, 2000.

[8] T. Arbogast, "A simplified dual-porosity model for two-phase flow," in Mathematical Modeling in Water Resources, T. F. Russell, R. E. Ewing, C. A. Brebbia, W. G. Gray, and G. F. Pindar, Eds., pp. 419-426, Computational Mechanics, Southampton, UK, 1992.

[9] B. Amaziane, J. P. Milišić, M. Panfilov, and L. Pankratov, "Generalized nonequilibrium capillary relations for two-phase flow through heterogeneous media," Physical Review E, vol. 85, Article ID 016304, 2012.

[10] M. Panfilov, "Homogenized model with capillary nonequilibrium for two-phase flow through highly heterogeneous porous media," Comptes Rendus de l'Académie des Sciences, Paris, Série IIB, vol. 322, pp. 195-202, 1996.

[11] A. Bourgeat and M. Panfilov, "Effective two-phase flow through highly heterogeneous porous media: capillary nonequilibrium effects," Computational Geosciences, vol. 2, no. 3, pp. 191-215, 1998.

[12] H. Salimi and J. Bruining, "Upscaling of fractured oil reservoirs using homogenization including non-equilibrium capillary pressure and relative permeability," Computational Geosciences, vol. 16, no. 2, pp. 367-389, 2012.

[13] A. Konyukhov and L. Pankratov, "Upscaling of an immiscible non-equilibrium two-phase flow in double porosity media," Applicable Analysis, vol. 95, no. 10, pp. 2300-2322, 2016.

[14] A. Konyukhov and L. Pankratov, "New non-equilibrium matrix imbibition equation for double porosity model," Comptes Rendus Mécanique, vol. 344, no. 7, pp. 510-520, 2016.

[15] X. Cao and I. S. Pop, "Two-phase porous media flows with dynamic capillary effects and hysteresis: uniqueness of weak solutions," Computers \& Mathematics with Applications, vol. 69, no. 7, pp. 688-695, 2015.

[16] X. Cao and I. S. Pop, "Degenerate two-phase porous media flow model with dynamic capillarity," Journal of Differential Equations, vol. 260, no. 3, pp. 2418-2456, 2016.

[17] J. Koch, A. Rätz, and B. Schweizer, “Two-phase flow equations with a dynamic capillary pressure," European Journal of Applied Mathematics, vol. 24, no. 1, pp. 49-75, 2013.

[18] A. Mikelić, "A global existence result for the equations describing unsaturated flow in porous media with dynamic capillary pressure," Journal of Differential Equations, vol. 248, no. 6, pp. 1561-1577, 2010. 
[19] C. Cuesta, C. J. van Duijn, and J. Hulshof, "Infiltration in porous media with dynamic capillary pressure: travelling waves," European Journal of Applied Mathematics, vol. 11, no. 4, pp. 381-397, 2000.

[20] C. J. van Duijn, Y. Fan, L. A. Peletier, and I. Pop, "Travelling wave solutions for degenerate pseudo-parabolic equations modelling two-phase flow in porous media," Nonlinear Analysis: Real World Applications, vol. 14, no. 3, pp. 1361-1383, 2013.

[21] K. Spayd and M. Shearer, "The Buckley-Leverett equation with dynamic capillary pressure," SIAM Journal on Applied Mathematics, vol. 71, no. 4, pp. 1088-1108, 2011.

[22] M. Peszyńska and S.-Y. Yi, "Numerical methods for unsaturated flow with dynamic capillary pressure in heterogeneous porous media," International Journal of Numerical Analysis and Modeling, vol. 5, pp. 126-149, 2008.

[23] M. Peszyńska, R. Showalter, and S.-Y. Yi, "Homogenization of a pseudoparabolic system," Applicable Analysis, vol. 88, no. 9, pp. 1265-1282, 2009.

[24] M. Jurak, L. Pankratov, and A. Vrbaški, "A fully homogenized model for incompressible two-phase flow in double porosity media," Applicable Analysis, vol. 95, no. 10, pp. 2280-2299, 2016.

[25] S. N. Antontsev, A. V. Kazhikhov, and V. N. Monakhov, Boundary Value Problems in Mechanics of Nonhomogeneous Fluids, North-Holland Publishing, Amsterdam, The Netherlands, 1990.

[26] J. Bear and Y. Bachmat, Introduction to Modeling of Transport Phenomena in Porous Media, Kluwer Academic Publishers, London, UK, 1991.

[27] G. Chavent and J. Jaffré, Mathematical Models and Finite Elements for Reservoir Simulation, North Holland, Amsterdam, The Netherlands, 1986.

[28] R. Helmig, Multiphase Flow and Transport Processes in the Subsurface, Springer, Berlin, Germany, 1997.

[29] G. I. Barenblatt and A. A. Gil'man, "Nonequilibrium counterflow capillary impregnation," Journal of Engineering Physics, vol. 52, no. 3, pp. 335-339, 1987.

[30] G. I. Barenblatt, J. Garcia-Azorero, A. De Pablo, and J. L. Vazquez, "Mathematical model of the non-equilibrium wateroil displacement in porous strata," Applicable Analysis. An International Journal, vol. 65, no. 1-2, pp. 19-45, 1997.

[31] G. I. Barenblatt, T. W. Patzek, and D. B. Silin, "The mathematical model of nonequilibrium effects in water-oil displacement," SPE Journal, vol. 8, no. 4, pp. 409-416, 2003.

[32] C. Cancès, "On the effects of discontinuous capillarities for immiscible two-phase flows in porous media made of several rock-types," Networks and Heterogeneous Media, vol. 5, no. 3, pp. 635-647, 2010.

[33] C. J. van Duijn, H. Eichel, R. Helmig, and I. S. Pop, "Effective equations for two-phase flow in porous media: the effect of trapping on the microscale," Transport in Porous Media, vol. 69, no. 3, pp. 411-428, 2007.

[34] T. Arbogast, J. Douglas, and U. Hornung, "Modelling of naturally fractured reservoirs by formal homogenization techniques," in Frontiers in Pure and Applied Mathematics, R. Dautray, Ed., pp. 1-19, Elsevier, Amsterdam, Netherlands, 1991.

[35] A. Bourgeat, S. Luckhaus, and A. Mikelić, "Convergence of the homogenization process for a double-porosity model of immiscible two-phase flow," SIAM Journal on Mathematical Analysis, vol. 27, no. 6, pp. 1520-1543, 1996.

[36] L.-M. Yeh, "Homogenization of two-phase flow in fractured media," Mathematical Models \& Methods in Applied Sciences, vol. 16, no. 10, pp. 1627-1651, 2006. 


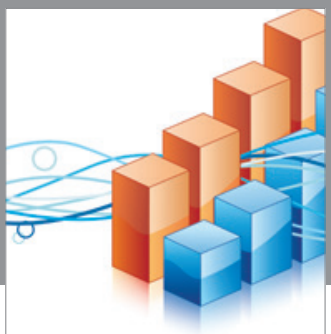

Advances in

Operations Research

vatem alat4

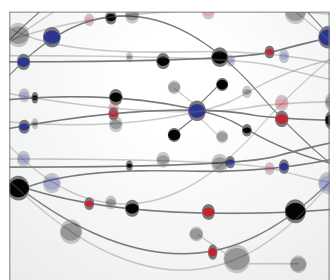

\section{The Scientific} World Journal
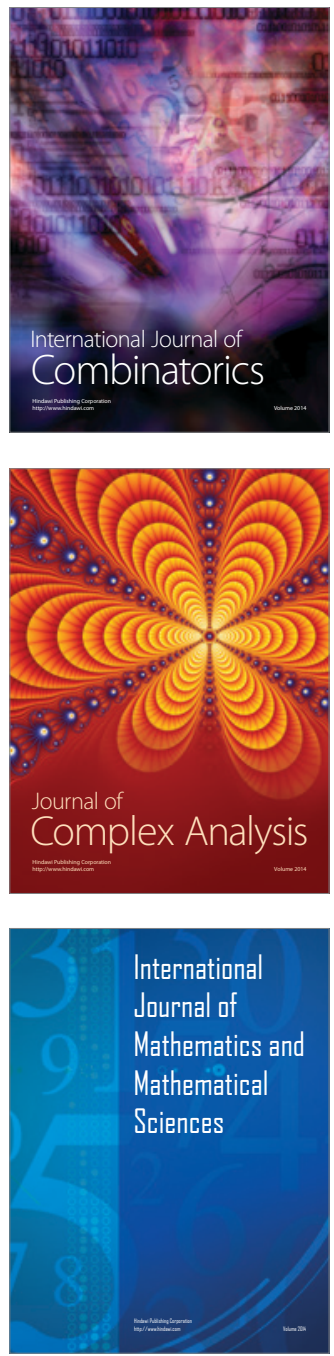
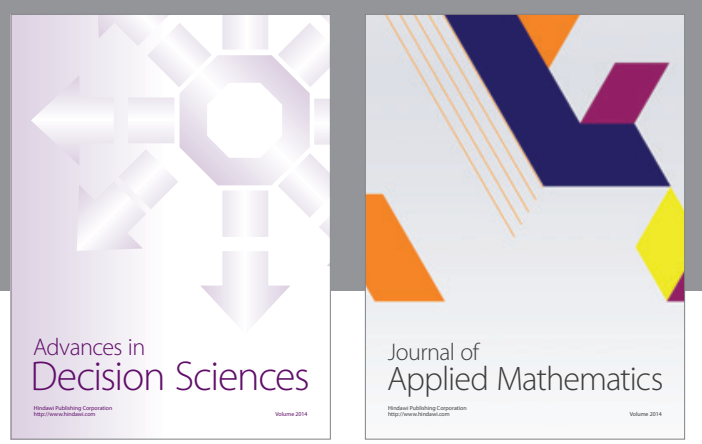

Algebra

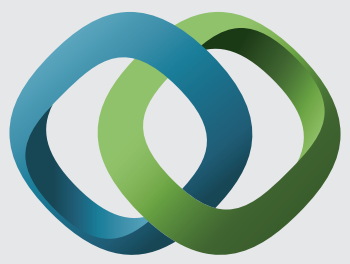

\section{Hindawi}

Submit your manuscripts at

http://www.hindawi.com
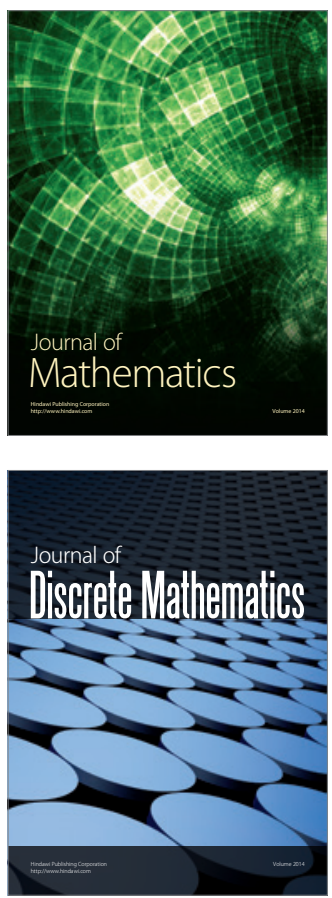

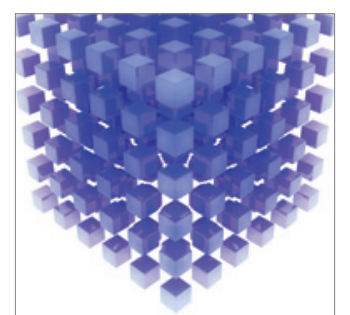

Mathematical Problems in Engineering
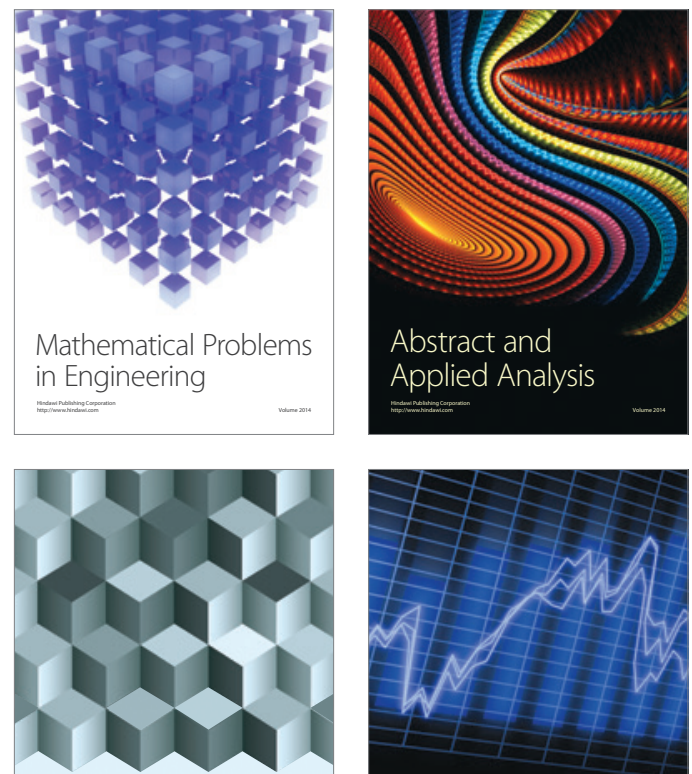

Journal of

Function Spaces

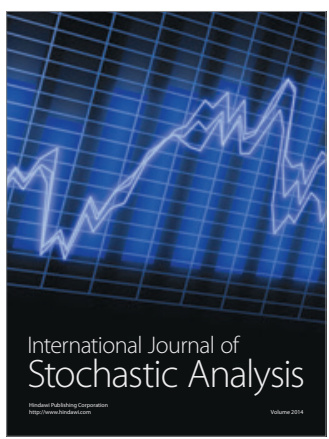

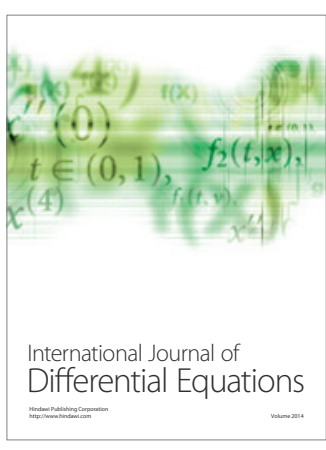
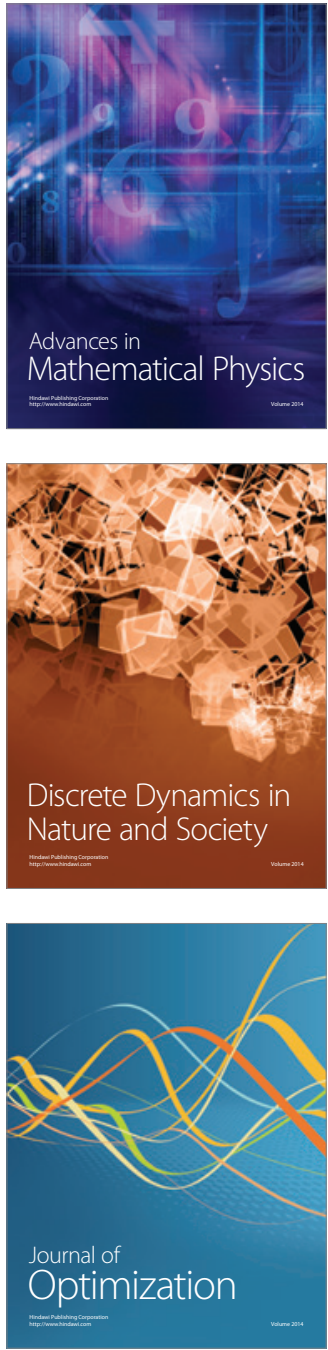